\title{
Fast GMTI Algorithm For Traffic Monitoring Based On A Priori Knowledge
}

\author{
Stefan V. Baumgartner, Graduate Student Member, IEEE, and Gerhard Krieger, Senior Member, IEEE
}

\begin{abstract}
In this paper, a fast a priori knowledge-based ground moving target indication and parameter estimation algorithm applicable to single- as well as to multichannel synthetic aperture airborne radar data is presented. The algorithm operates directly on range-compressed data. Only the intersection points of the moving vehicle signals with the $a$ priori known road axes, which are mapped into the range-compressed data array, are evaluated. For moving vehicle detection and parameter estimation, basically only a single 1-D fast Fourier transformation has to be performed for each considered road point. Hence, the required computational power is low, and the algorithm is well suited for real-time traffic monitoring applications. The proposed algorithm enables the estimation of the position and velocity vectors of detected moving vehicles independent of the number of channels. A single-channel synthetic aperture radar system may be sufficient in case of fast moving vehicles. The paper includes a detailed performance assessment together with experimental results that demonstrate the applicability in a real-world scenario.
\end{abstract}

Index Terms-Pulse Doppler radar, radar signal processing, road vehicle detection, synthetic aperture radar (SAR).

\section{INTRODUCTION}

$\mathbf{N}$ OWADAYS, a lot of motorways are equipped with sensors to monitor the actual traffic situation with the aim to ensure mobility and to increase the safety of the road users. Unfortunately, such detailed traffic information is missing outside the major motorways due to the lack of sensor installations. Radars flying at high altitudes provide an elegant solution to fill this gap, particularly if this information is required only on a nonregular basis as in the case of special events or catastrophes. Such a radar system has the challenging task to acquire, process and deliver the relevant traffic products to a dedicated traffic management center in real time. Synthetic aperture radar (SAR) processing and ground moving target indication (GMTI) have to be carried out directly on-board the radar platform. Due to bandwidth limitations, only the relevant traffic data can be transmitted to a ground station using a conventional data link.

From the operational point of view, the use of an aircraft as platform instead of a single spacecraft provides more flexibility and allows for shorter revisit and longer observation times at the cost of a reduced spatial coverage. We see, however, a great potential for future high altitude platforms flying in the upper

Manuscript received March 24, 2011; revised September 8, 2011 and December 9, 2011; accepted February 24, 2012. Date of publication May 14, 2012; date of current version October 24, 2012

The authors are with the Radar Concepts Department of the Microwaves and Radar Institute, German Aerospace Center (DLR), 82234 Oberpfaffenhofen, Germany (e-mail: stefan.baumgartner@dlr.de; gerhard.krieger@dlr.de).

Color versions of one or more of the figures in this paper are available online at http://ieeexplore.iee.org.

Digital Object Identifier 10.1109/TGRS.2012.2193133 troposphere or even in the stratosphere [1]. These platforms are not only well suited for the real-time monitoring of selected areas, but they can also accommodate large antennas which provide an excellent signal-to-noise ratio (SNR).

Principally already existing GMTI techniques, most of which originated in the military field, can be used for moving vehicle detection and parameter estimation. Examples are the algorithms presented in [2]-[7]. However, most of these algorithms require large computing power and, particularly if the computation should be performed in real time, the system complexity and the costs will become enormous. By incorporating the a priori known road network into the detection stage of the GMTI algorithm and by ignoring off-road moving vehicles, the system complexity as well as the computational load and the costs can be reduced significantly. Furthermore, for many traffic monitoring applications, target tracking (which often is necessary if space-time adaptive processing (STAP) algorithms are used [7]) is not required, and a snapshot of the actual traffic situation is sufficient.

The idea of using a road network is not new, but up to now, such a road network mainly was used together with displacement-based GMTI algorithms [8]. These algorithms measure the azimuth displacements of moving vehicles, occurring in SAR images. The required preprocessing is time consuming since in general, SAR images have to be generated taking into account the full bandwidth given by the pulse repetition frequency (PRF). The across-track velocities of the vehicles are then computed by exploiting the relationship between azimuth displacement and across-track velocity.

Our proposed algorithm is mainly designed for real-time airborne or near-space vehicle-born traffic monitoring applications. Since SNR is in general quite large in this case, SAR focusing often is not required for GMTI. Therefore, the algorithm operates directly on single- or multichannel range-compressed airborne SAR data. The geocoded position of each detected moving vehicle is obtained from the intersection of the road axis with the range-compressed vehicle signal. Motion parameter estimation is done by estimating the Doppler frequency of the signal at the road intersection. The parameters absolute velocity, heading, and geocoded position can be estimated with high accuracy.

The algorithm in its actual form is not envisaged for spaceborne systems, which in general suffer from the low SNR values of the range-compressed data. An extension would be possible if the range cell migration of the moving vehicle signal is considered during processing and a proper reference function is used for focusing, which has the potential to increase the SNR as well as the signal-to-clutter plus noise ratio (SCNR) 

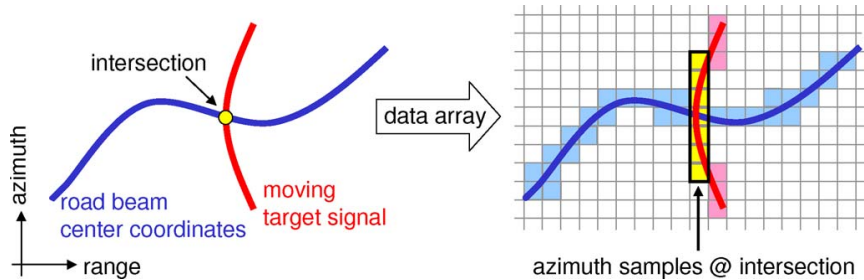

Fig. 1. Principle of the proposed GMTI algorithm. The intersection of the moving vehicle signal with the road corresponds to the vehicle's beam center position.

significantly. For this task in principle, a matched-filter bank can be used [5], [9]. However, further investigations are necessary which are beyond the scope of this paper.

The paper is organized as follows. In Section II, the proposed algorithm is introduced and explained in detail. A comprehensive discussion about the achievable performance is given in Section III, and before the paper concludes with Section V, experimental results are presented in Section IV.

The actual paper is a comprehensive extension of our conference papers [10], [11] presented at the European Conference on Synthetic Aperture Radar and at the IEEE International Geoscience and Remote Sensing Symposium in 2010.

\section{Algorithm}

\section{A. Principle}

As a first step, the a priori known road axis of interest is mapped into the range-compressed SAR data array. The required coordinate transformation, which is the heart of the proposed algorithm, is performed in such a way that the geographical coordinates of each road point are transformed to corresponding azimuth beam center coordinates ${ }^{1}$ in the range/azimuth plane. The azimuth beam center position of the detected moving vehicle is then directly given by the intersection of the vehicle signal with the mapped road point (cf. Fig. 1 left).

As a consequence of the mapping, the geographical coordinates of the road point and, hence, the coordinates of the detected vehicle moving on this road point at beam center time $t_{\mathrm{bc}}$ are known, so that no further geocoding is required. For moving vehicle detection and motion parameter estimation, only a few azimuth samples around the intersection point are taken (cf. Fig. 1 right) and transformed into the Doppler domain using a fast Fourier transformation (FFT). Due to the small number of azimuth samples, the signal phase is more or less linear over the short observation time, so that the vehicle appears as a sharp peak in the Doppler domain. For detection, the peak amplitude is compared to a certain threshold and for motion parameter estimation the Doppler shift $f_{\mathrm{DC}}$ of the signal peak is exploited (cf. Section II-D).

\section{B. Structure of the Algorithm}

In Fig. 2, the flow chart of the proposed a priori knowledgebased GMTI algorithm is shown exemplarily for a dual-channel

\footnotetext{
${ }^{1}$ With "azimuth beam center" the center of the antenna beam in azimuth direction is meant.
}

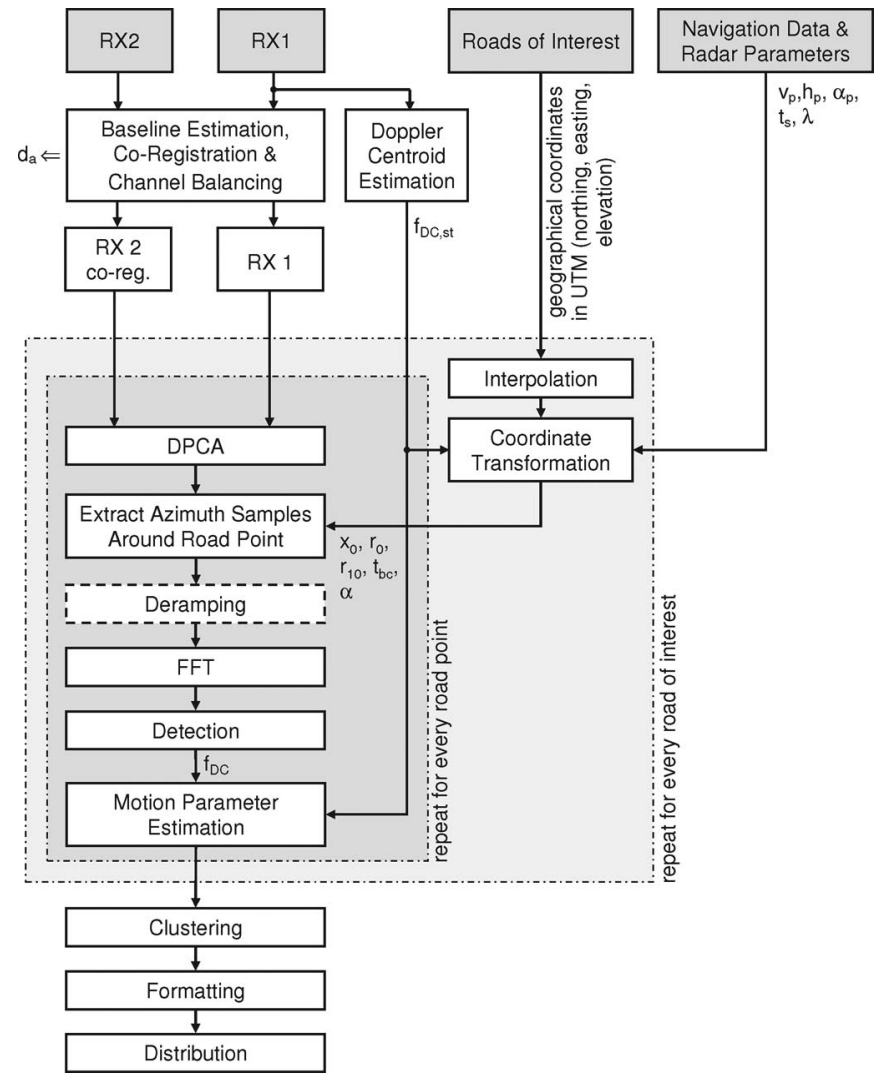

Fig. 2. Simplified flow chart of the proposed a priori knowledge-based GMTI algorithm for a dual-channel SAR system (the "deramping" block is optional).

system. RX1 and RX2 are the range-compressed SAR data acquired with the two channels. After baseline estimation, channel coregistration and digital channel balancing [12], [13], clutter suppression is performed using the displaced phase center antenna (DPCA) technique [14].

The geographical coordinates as well as the elevations of the roads of interest, on which the road traffic should be monitored, are directly obtained from a road database. Since the coordinate transformation (cf. Section II-C) is done in a Cartesian coordinate system, the geographical road coordinates should be in Universal Transverse Mercator (UTM) projection (UTM coordinates themselves are Cartesian coordinates). In general, before performing the coordinate transformation, an interpolation of the geographical road coordinates is necessary for avoiding gaps in the range/azimuth plane or SAR data array, respectively.

Around each road point in the range/azimuth plane, some azimuth samples are taken directly from the DPCA data. Depending on the desired performance a deramping operation, where the azimuth chirp of the signal is removed, can be performed optionally before transforming the azimuth samples into the Doppler domain using a FFT (cf. Section III-C and D). Detection is done by applying a certain threshold directly in the Doppler domain. Each detected signal peak has a certain Doppler shift $f_{\mathrm{DC}}$ and corresponds to a certain moving vehicle. Motion parameter estimation is then performed for each detected vehicle by exploiting the estimated Doppler shift and the known position parameters of the observed road point 


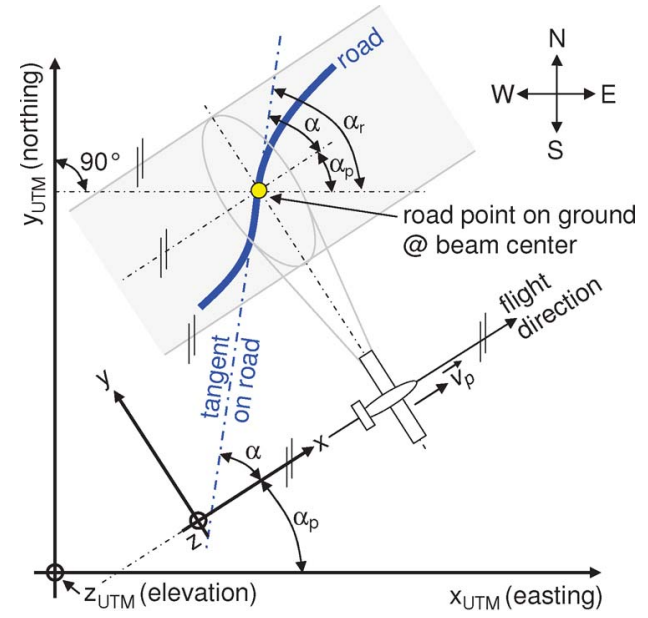

Fig. 3. Relation between the geographical Cartesian UTM coordinate system $\left\{x_{\mathrm{UTM}}, y_{\mathrm{UTM}}, z_{\mathrm{UTM}}\right\}$ and local Cartesian coordinate system $\{x, y, z\}$.

(cf. Section II-D). For each road of interest, the same procedure has to be repeated. Before formatting and distributing the data for example to a traffic management center, a clustering operation is performed where multiple detections of one and the same vehicle are merged to only one physical vehicle.

It has to be mentioned that the algorithm sketched in Fig. 2 also can be used for single-channel systems by just omitting the stages "baseline estimation, coregistration, and channel balancing" and "DPCA." With single-channel systems, only fast moving vehicles falling outside the clutter band are detectable, but for these fast vehicles, the parameters absolute velocity, heading, and geocoded position can be estimated with high accuracy.

Furthermore, instead of having only two channels and using DPCA for clutter suppression, also several channels and more sophisticated clutter suppression techniques like STAP [7] can be used, at the cost of higher computational demands and increased system complexity. Nevertheless, to keep the paper clear, only the one- and two-channel cases are discussed.

In the following two subsections, the coordinate transformation and the motion parameter estimation procedure are explained in detail.

\section{Coordinate Transformation}

The relation between the global Cartesian UTM coordinate system $\left\{x_{\mathrm{UTM}}, y_{\mathrm{UTM}}, z_{\mathrm{UTM}}\right\}$ and the local Cartesian coordinate system $\{x, y, z\}$ relevant for GMTI processing is sketched in Fig. 3. The $x$-axis of the local coordinate system is defined by the platform velocity vector $\vec{v}_{\mathrm{p}}$, which is assumed to be constant during the observation interval. If the platform moves at constant altitude $h_{\mathrm{p}}$, the local coordinate system is more or less a rotation and translation of the global UTM coordinate system, and the $z$-axis is parallel to the $z_{\mathrm{UTM}}$-axis.

In practice, it cannot be ensured that the antenna squint angle $\psi$, and hence the Doppler centroid $f_{\mathrm{DC}, \text { st }}$ of the stationary scene are negligibly small. Although the positions of the road points in the local coordinate system shown in Fig. 3 are independent of the actual squint angle, a squinted geometry (cf. Fig. 4 right) has to be considered for mapping each road point from the

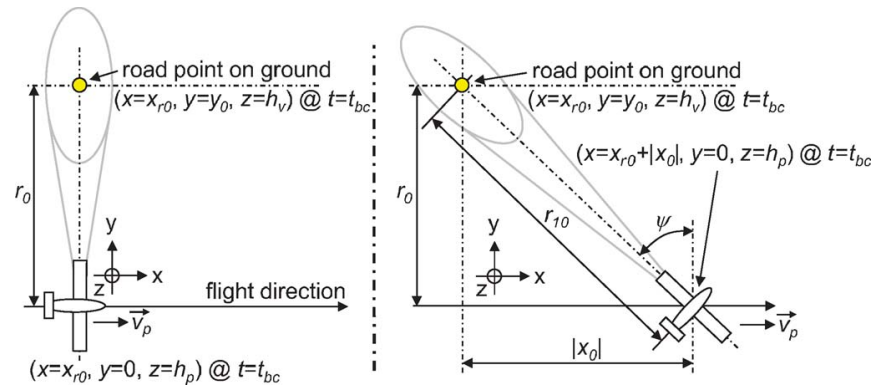

Fig. 4. Nonsquinted (left) and squinted (right) data acquisition geometry. The $x$-axis is parallel to the flight direction given by $\vec{v}_{p}$. The azimuth beam center positions of the road point on ground are marked with a circle.

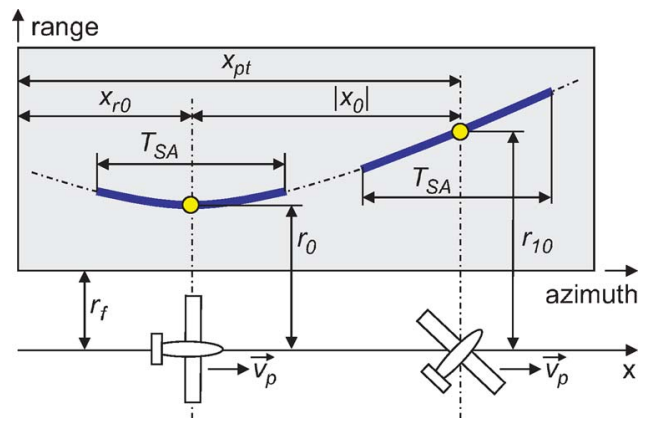

Fig. 5. SAR data array containing the acquired range-compressed data of a single road point in the nonsquinted (left) and squinted (right) case. The beam center positions of the road point are marked with a circle.

local $\{x, y, z\}$ coordinate system into the range/azimuth plane or SAR data array (cf. Fig. 5), respectively. In the following derivation for simplicity (but without loss of generality) only one single, common local $x$-axis is used. The necessary translation between the flight path given by the local $x$-axis and the azimuth axis is considered by the range and squint angledependent azimuth shift $x_{0}$, which is explained later in this section.

In Fig. 5, it is shown how the received nonsquinted and squinted data of one and the same stationary road point are stored in the range-compressed SAR data array. $T_{\mathrm{SA}}$ denotes the maximum illumination time or synthetic aperture time, $x_{\mathrm{r} 0}$ is the azimuth position of the road point at minimum range $r_{0}$, $x_{0}$ is the azimuth offset owing to the squint angle $\psi, x_{\mathrm{pt}}$ is the position of the road point if the squint angle is considered, and $r_{10}$ is the azimuth beam center range (i.e., the range between the antenna phase center on-board the platform and the road point on the ground located at the azimuth center of the illuminating antenna beam).

By decomposing the range vector $\vec{r}$ (pointing from the RX/TX antenna phase center to the stationary road point on ground) into a component parallel and a component perpendicular to the flight direction, the vectors $\vec{x}_{\mathrm{r} 0}$ and $\vec{r}_{0}$ are obtained (note that these vectors are given in the $\left\{x_{\mathrm{UTM}}, y_{\mathrm{UTM}}, z_{\mathrm{UTM}}\right\}$ coordinate system)

$$
\vec{x}_{\mathrm{r} 0}\left(t=t_{\mathrm{s}}\right)=\left\langle\vec{v}_{\mathrm{p}}, \vec{r}\left(t=t_{\mathrm{s}}\right)\right\rangle \frac{\vec{v}_{\mathrm{p}}}{\left\|\vec{v}_{\mathrm{p}}\right\|^{2}}
$$

where $t_{\mathrm{s}}$ is the absolute start time of data acquisition obtained from the radar control unit, $\langle$.$\rangle is the inner product, and \|$. 
is the $L_{2}$ norm. The vectors $\vec{v}_{\mathrm{p}}$ and $\vec{r}$ can be computed using the known UTM coordinates of the stationary road point and the radar platform at any time instant (i.e., $\vec{r}(t)=\vec{r}_{\text {roadpoint }}-$ $\vec{r}_{\text {platform }}(t)$, where $\vec{r}_{\text {roadpoint }}$ is the UTM position of the road point obtained from the road database, and $\vec{r}_{\text {platform }}$ is the UTM position of the radar platform obtained from the onboard GPS system; $\vec{v}_{\mathrm{p}}$ is the time derivative of $\vec{r}_{\text {platform }}(t)$ which is assumed to be constant). The minimum range is then given by

$$
r_{0}=\left\|\vec{r}\left(t=t_{\mathrm{s}}\right)-\vec{x}_{\mathrm{r} 0}\left(t=t_{\mathrm{s}}\right)\right\| .
$$

The $x$-position of the stationary road point in the local $\{x, y, z\}$ coordinate system can be written as

$$
x_{\mathrm{r} 0}=\left\langle\frac{\vec{v}_{\mathrm{p}}}{\left\|\vec{v}_{\mathrm{p}}\right\|}, \frac{\vec{x}_{\mathrm{r} 0}\left(t=t_{\mathrm{s}}\right)}{\left\|\vec{x}_{\mathrm{r} 0}\left(t=t_{\mathrm{s}}\right)\right\|}\right\rangle\left\|\vec{x}_{\mathrm{r} 0}\left(t=t_{\mathrm{s}}\right)\right\|
$$

where the term $\langle$.$\rangle is necessary for determining the correct sign$ (it either gives +1 or -1 ). The azimuth position of this stationary road point within the SAR data array can be computed by the following equation:

$$
x_{\mathrm{pt}}=x_{\mathrm{r} 0}-x_{0} .
$$

The azimuth offset $x_{0}$ caused by the squint angle $\psi$ can be computed as

$$
x_{0}=r_{0} \tan \psi
$$

where the squint angle is given by

$$
\psi=\arcsin \left(\frac{\lambda f_{\mathrm{DC}, \mathrm{st}}}{2 v_{\mathrm{p}}}\right)=\arccos \left(\frac{r_{0}}{r_{10}}\right) .
$$

In the previous equation, $\lambda$ is the radar wavelength, $f_{\mathrm{DC}, \mathrm{st}}$ is the Doppler centroid of the clutter, which can directly be estimated from the range-compressed data of a single channel, and $v_{\mathrm{p}}$ is the magnitude of the platform velocity vector $\vec{v}_{\mathrm{p}}$. Please note that both $\psi$ and $f_{\mathrm{DC}, \mathrm{st}}$ are zero if the antenna points exactly perpendicular to the $x$-axis. The positive measurement direction of $\psi$ is clockwise, the negative sign in front of $x_{0}$ in (4) is necessary for computing the correct azimuth positions.

In general, the Doppler centroid $f_{\mathrm{DC}, \text { st }}$ and, hence, the squint angle $\psi$ are range dependent. Ignoring this range dependence, by considering only the average of the Doppler centroid, the road point of interest will not be mapped at every range exactly at the azimuth beam center position, but slightly shifted. This fact is discussed in Section III-A.

The azimuth beam center range can then be computed as

$$
r_{10}=\frac{r_{0}}{\cos \psi} .
$$

The absolute beam center time (mapping time; i.e., the time when the road point is observed by the azimuth beam center of the antenna) of the road point or the detected vehicle moving on this road point, respectively, is given by

$$
t_{\mathrm{bc}}=t_{\mathrm{s}}+\frac{x_{\mathrm{pt}}}{v_{\mathrm{p}}} .
$$

The values of $x_{\mathrm{pt}}$ and $r_{10}$ can be transformed to range and azimuth sample numbers by considering the PRF and the range sampling frequency $f_{\mathrm{r}}$ (cf. Fig. 5)

$$
\begin{aligned}
& x_{\mathrm{pt}, \mathrm{S}}=\text { round }\left[x_{\mathrm{pt}} \frac{\mathrm{PRF}}{v_{\mathrm{p}}}\right] \\
& r_{10, \mathrm{~S}}=\text { round }\left[\left(r_{10}-r_{\mathrm{f}}\right) \frac{2 \mathrm{f}_{\mathrm{r}}}{\mathrm{c}}\right]
\end{aligned}
$$

where round[.] is a rounding operation to nearest integer, $\mathrm{c}$ is the speed of light and $r_{\mathrm{f}}$ is the known range corresponding to the first range bin stored in the SAR data array (cf. Fig. 5).

The road angle $\alpha_{\mathrm{r}}$ corresponding to the observed road point can be computed considering the neighboring road points from the road database. ${ }^{2}$ The road angle with respect to the flight path or the local $x$-axis, respectively, is then given by (cf. Fig. 3)

$$
\alpha=\alpha_{\mathrm{r}}-\alpha_{\mathrm{p}}
$$

where $\alpha_{\mathrm{p}}$ is the track angle of the platform with respect to the $x_{\mathrm{UTM}}$-axis of the UTM coordinate system given as

$$
\alpha_{\mathrm{p}}=\arccos \left\langle\left(\begin{array}{l}
1 \\
0 \\
0
\end{array}\right), \frac{\vec{v}_{\mathrm{p}}}{\left\|\vec{v}_{\mathrm{p}}\right\|}\right\rangle .
$$

\section{Motion Parameter Estimation}

In the following derivation for simplicity, only a single antenna and, thus, a single-channel moving vehicle signal is considered. This is sufficient, because after clutter suppression, the Doppler shift and slope (which are exploited for motion parameter estimation) of a multichannel signal do not significantly differ from those of a single-channel signal as long as the observation time lag caused by the along-track baseline is small (for typical airborne systems the time lag is in the order of a millisecond or even smaller) [15].

The radar platform moves only along the $x$-axis of the local coordinate system, so that its motion equations can be written as

$$
\begin{aligned}
& x_{\mathrm{p}}=v_{\mathrm{p}}\left(t-t_{\mathrm{s}}\right) \\
& y_{\mathrm{p}}=0 \\
& z_{\mathrm{p}}=h_{\mathrm{p}} .
\end{aligned}
$$

Furthermore, the motion equations of a moving vehicle under the assumption that it moves linear with constant acceleration at constant altitude $h_{\mathrm{v}}$ in a plane parallel to the $x-y$-plane can then be written as

$$
\begin{aligned}
& x_{\mathrm{v}}=x_{\mathrm{r} 0}+v_{0} \cdot\left(t-t_{\mathrm{bc}}\right) \cos \alpha_{\mathrm{v}}+\frac{1}{2} a \cdot\left(t-t_{\mathrm{bc}}\right)^{2} \cos \alpha_{\mathrm{v}} \\
& y_{\mathrm{v}}=y_{0}+v_{0} \cdot\left(t-t_{\mathrm{bc}}\right) \sin \alpha_{\mathrm{v}}+\frac{1}{2} a \cdot\left(t-t_{\mathrm{bc}}\right)^{2} \sin \alpha_{\mathrm{v}} \\
& z_{\mathrm{v}}=h_{\mathrm{v}}
\end{aligned}
$$

\footnotetext{
${ }^{2}$ The road angle $\alpha_{\mathrm{r}}$ is measured from the $x_{\mathrm{UTM}}$-axis. The positive counting direction is counterclockwise.
} 
where $v_{0}$ is the magnitude of the vehicle velocity at beam center time $t_{\mathrm{bc}}, a$ is its constant acceleration, and $\alpha_{\mathrm{v}}$ is the moving direction with respect to the flight path. Note that the moving direction $\alpha_{\mathrm{v}}$ is assumed to correspond to the a priori known road angle $\alpha$. It either can be $\alpha_{\mathrm{v}}=\alpha$ or $\alpha_{\mathrm{v}}=\alpha-180^{\circ}$ [from the parameter estimation point of view this ambiguity can be resolved with (26)]. The across-track position of the target at $t=t_{\mathrm{bc}}$ is denoted as $y_{0}$ and given as

$$
y_{0}=\sqrt{r_{0}^{2}-\Delta h^{2}}
$$

where $\Delta h=h_{\mathrm{v}}-h_{\mathrm{p}}$ is the altitude difference between the moving vehicle on the road point of interest and the radar platform. The distance from the transmit antenna to the vehicle is then

$$
r(t)=\sqrt{\left(x_{\mathrm{v}}-x_{\mathrm{p}}\right)^{2}+y_{\mathrm{v}}^{2}+\Delta h^{2}}
$$

The range-compressed moving vehicle signal received by the radar can be modeled as (one single TX/RX antenna assumed)

$$
s(t)=A\left(t-t_{\mathrm{bc}}\right) \exp \left\{-j \frac{4 \pi}{\lambda} r(t)\right\}
$$

where $A\left(t-t_{\mathrm{bc}}\right)$ includes the two-way antenna pattern, the target reflectivity, and propagation losses. After performing a second-order Taylor approximation around $t=t_{\mathrm{bc}}$ and some substitutions, the range $r(t)$ given in (20) can be written as [16]

$$
r(t) \cong r_{10}-\frac{\lambda}{2} f_{\mathrm{DC}}\left(t-t_{\mathrm{bc}}\right)-\frac{\lambda}{4} k_{\mathrm{a}}\left(t-t_{\mathrm{bc}}\right)^{2}
$$

where $f_{\mathrm{DC}}$ is the total Doppler shift of the received signal due to squint and target motion, and $k_{\mathrm{a}}$ is the Doppler slope. The corresponding equations are

$$
\begin{gathered}
f_{\mathrm{DC}}=-\frac{2}{\lambda r_{10}}\left[x_{0}\left(v_{0} \cos \alpha_{\mathrm{v}}-v_{\mathrm{p}}\right)+y_{0} v_{0} \sin \alpha_{\mathrm{v}}\right] \\
k_{\mathrm{a}}=-\frac{2}{\lambda r_{10}}\left\{x_{0} a \cos \alpha_{\mathrm{v}}+\left(v_{0} \cos \alpha_{\mathrm{v}}-v_{\mathrm{p}}\right)^{2}\right. \\
+y_{0} a \sin \alpha_{\mathrm{v}}+v_{0}^{2} \sin ^{2} \alpha_{\mathrm{v}} \\
-\frac{1}{r_{10}^{2}}\left[x_{0}\left(v_{0} \cos \alpha_{\mathrm{v}}-v_{\mathrm{p}}\right)\right. \\
\left.\left.+y_{0} v_{0} \sin \alpha_{\mathrm{v}}\right]^{2}\right\}
\end{gathered}
$$

The total Doppler shift $f_{\mathrm{DC}}$ can be estimated by peak detection after transforming the azimuth samples around the intersection point with the road (cf. Fig. 1 right) into the Doppler domain using a FFT. The optional application of the "deramping" block shown in Fig. 2 before performing the FFT influences the estimation accuracy (cf. Section III-C and D), but not the estimation principle.

The absolute beam center velocity $v_{0}$ of the moving vehicle can be computed using (23), (5), and (6)

$$
v_{0}=\left|\frac{\lambda r_{10}\left(f_{\mathrm{DC}, \mathrm{st}}-f_{\mathrm{DC}}\right)}{2\left(x_{0} \cos \alpha_{\mathrm{v}}+y_{0} \sin \alpha_{\mathrm{v}}\right)}\right|=\left|v_{\mathrm{abs}}\right| .
$$

Furthermore, the "true" heading of the moving vehicle is given as (remember that the vehicle can move either in or against the known road direction)

$$
\alpha_{\mathrm{v}, \text { true }}=\left\{\begin{aligned}
\alpha_{\mathrm{v}} & \text { if } \operatorname{sgn}\left(v_{\text {abs }}\right)=+1 \\
\alpha_{\mathrm{v}}-180^{\circ} & \text { if } \operatorname{sgn}\left(v_{\text {abs }}\right)=-1
\end{aligned}\right.
$$

where $\operatorname{sgn}($.$) is the signum function.$

The heading with respect to the $x$-axis of the UTM coordinate system is then $\alpha_{\mathrm{v}, \mathrm{UTM}}=\alpha_{\mathrm{v} \text {,true }}+\alpha_{\mathrm{p}}$, and the geographical heading $\left(0^{\circ}=N, 90^{\circ}=E, 180^{\circ}=S, 270^{\circ}=W\right)$ can be expressed as

$$
\alpha_{\mathrm{v}, \text { geo }}=\left(90^{\circ}-\alpha_{\mathrm{v}, \mathrm{UTM}}+\beta\right) \bmod 360^{\circ}
$$

where mod is the modulo operation and $\beta$ the meridian convergence.

Assuming that also the Doppler slope $k_{\mathrm{a}}$ can be estimated by using for example a matched-filter bank [16] or "adaptive deramping" (cf. Section III-D), the acceleration of the moving vehicle can be computed as

$$
\begin{aligned}
a= & \frac{1}{x_{0} \cos \alpha_{\mathrm{v}, \text { true }}+y_{0} \sin \alpha_{\mathrm{v}, \text { true }}} \\
\times\left\{-\frac{1}{2} k_{\mathrm{a}} \lambda r_{10}+\frac{1}{r_{10}^{2}}[\right. & x_{0}\left(v_{0} \cos \alpha_{\mathrm{v}, \text { true }}-v_{\mathrm{p}}\right) \\
& \left.+y_{0} v_{0} \sin \alpha_{\mathrm{v}, \text { true }}\right]^{2} \\
& \left.-v_{0}^{2} \sin ^{2} \alpha_{\mathrm{v}, \text { true }}-\left(v_{0} \cos \alpha_{\mathrm{v}, \text { true }}-v_{\mathrm{p}}\right)^{2}\right\} .
\end{aligned}
$$

\section{Performance Assessment}

In this section, we investigate the parameter estimation performance of the new GMTI algorithm. Before analyzing the impact of different error sources, some basic assumptions shall be clarified: in reality, the radar cross section (RCS) of a vehicle strongly depends on the aspect angle. Even a small change in aspect angle may result in a severe RCS change of several $\mathrm{dBm}^{2}$, as shown by simulations in [17] and by real measurements in [18]. In principle, an RCS change might also result in a phase change of the signal, and the phase change may have some influence on the resulting Doppler shift, although such an effect has not been reported in the aforementioned references. In the following theoretical investigations, it is assumed that the moving vehicle can be considered as a point-like target with constant and aspect angle-independent RCS. This assumption is also justified by the fact that our algorithm only uses a few azimuth samples corresponding to a very small aspect angle change and, hence, to a rather small RCS change. A consideration of phase changes owing to RCS fluctuations would require a more sophisticated moving vehicle signal model and simulations, which are beyond the scope of this paper.

The theoretical detection performance, for example as a function of line-of-sight velocity and SCNR, is also not investigated. For assessing the detection performance from a theoretical point of view, the methods presented in [19], [20] can be used. It has to be pointed out that the detection performance 
of the proposed algorithm depends mainly on the preprocessing used for clutter suppression (e.g., no preprocessing and no clutter suppression of single-channel data, application of DPCA or STAP for multichannel data) and not directly on the proposed algorithm itself. Therefore, the error analysis given in the following subsections should only show the performance limits which are relevant for the proposed algorithm, under the assumption that the vehicle already has been detected.

\section{A. Coordinate Transformation Errors}

Assuming that the geographical positions and elevations of the radar platform as well as of the road axis are known with high accuracy, only two potential error sources have to be considered at the coordinate transformation:

- Inaccurate and range-dependent squint angle $\psi$, which is computed from the estimate of the Doppler centroid $f_{\mathrm{DC}, \mathrm{st}}$;

- Difference between the true vehicle position and the road axis position.

1) Inaccurate Squint Angle: For the proposed algorithm, the squint angle $\psi$ is assumed to be constant, but in reality it changes over range. It can be shown that due to a squint angle change $\Delta \psi$, the vehicle is seen at a different time $t_{\mathrm{bc}}+\Delta t$. The vehicle will be detected at this "new" time at its "new and correct" position and with its "new and correct" velocity. Thus, a squint angle change results neither in a position nor in a velocity error. During $\Delta t$, the vehicle has moved to the new positions

$$
\begin{aligned}
& x_{\mathrm{v}, \text { new }}=x_{\mathrm{v}}+v_{0} \Delta t \cos \alpha_{\mathrm{v}}+\frac{1}{2} a \Delta t^{2} \cos \alpha_{\mathrm{v}} \\
& y_{\mathrm{v}, \text { new }}=y_{\mathrm{v}}+v_{0} \Delta t \sin \alpha_{\mathrm{v}}+\frac{1}{2} a \Delta t^{2} \sin \alpha_{\mathrm{v}} .
\end{aligned}
$$

The new velocity is then

$$
v_{0, \text { new }}=v_{0}+a \Delta t .
$$

For small squint angle changes $\Delta \psi$ and, hence, small time differences $\Delta t$, the acceleration $a$ can be neglected (for a time difference of $1 \mathrm{~s}$, an acceleration of $0.5 \mathrm{~m} / \mathrm{s}^{2}$ results in a velocity change of $1.8 \mathrm{~km} / \mathrm{h}$ and in an additional position change of $0.25 \mathrm{~m}$ ). The time difference as a function of velocity and squint angle change can then be approximated as

$$
\Delta t \cong \frac{\sqrt{y_{0}^{2}+\Delta h^{2}}}{v_{0} \cos \alpha_{\mathrm{v}}-v_{\mathrm{p}}} \tan \Delta \psi .
$$

By applying different squint angle assumptions during the coordinate transformation, one and the same vehicle might be detected at different times and positions. This might improve the detection probability significantly, particularly under the viewpoint that in reality, the RCS of a vehicle strongly depends on the aspect angle [17], [18]. Multiple detections can easily be clustered to one physical target knowing the detected positions, velocities, and time differences. Furthermore, a kind of vehicle tracking is possible, but this is not a topic of this paper.
TABLE I

SYSTEM AND GEOMETRY PARAMETERS

\begin{tabular}{lcc}
\hline Speed of light & $\mathrm{c}$ & $2.9979 \cdot 10^{8} \mathrm{~m} / \mathrm{s}$ \\
Platform velocity & $v_{p}$ & $90 \mathrm{~m} / \mathrm{s}$ \\
Pulse repetition frequency & $\mathrm{PRF}$ & $5000 \mathrm{~Hz}$ \\
Range sampling frequency & $\mathrm{f}_{\mathrm{r}}$ & $100 \mathrm{MHz}$ \\
Wavelength & $\lambda$ & $0.03125 \mathrm{~m}$ \\
Antenna length & $L_{\mathrm{a}}$ & $0.2 \mathrm{~m}$ \\
Altitude difference & $|\Delta h|$ & $2200 \mathrm{~m}$ \\
\hline
\end{tabular}

2) Road Axis Position: Each road or lane, respectively, is represented as a single road axis in the road database. Since the road width is in the order of a few meters, a vehicle does not necessarily move exactly on the road axis. Thus, the position difference between the true vehicle position and the intersection of the moving vehicle signal with the road axis is an inherent error of the proposed algorithm. This error is in practice maybe in the order of 5 to $20 \mathrm{~m}$, depending mainly on the road width and on the accuracy of the geographical positions of the road axis. As a consequence, the estimated Doppler shift at the intersection does not correspond to the Doppler shift at the true vehicle position. Assuming that the road point positions $x_{0}$ and $y_{0}$ are erroneous, by using the laws of error propagation, the error of the Doppler shift can be expressed as (again the acceleration has been neglected since its influence on the Doppler shift for small changes of $x_{0}$ and $y_{0}$ is not significant)

$$
\sigma_{f_{\mathrm{DC}}}=\sqrt{\left(\frac{\partial f_{\mathrm{DC}}}{\partial x_{0}}\right)^{2} \sigma_{x_{0}}^{2}+\left(\frac{\partial f_{\mathrm{DC}}}{\partial y_{0}}\right)^{2} \sigma_{y_{0}}^{2}}
$$

with

$$
\begin{aligned}
\frac{\partial f_{\mathrm{DC}}}{\partial x_{0}} & =\frac{2\left(v_{0} \cos \alpha_{v}-v_{\mathrm{p}}\right)}{\lambda r_{10}}\left(\frac{x_{0}^{2}}{r_{10}^{2}}-1\right) \\
\frac{\partial f_{\mathrm{DC}}}{\partial y_{0}} & =\frac{2 v_{0} \sin \alpha_{v}}{\lambda r_{10}}\left(\frac{y_{0}^{2}}{r_{10}^{2}}-1\right)
\end{aligned}
$$

where $\sigma_{x_{0}}$ and $\sigma_{y_{0}}$ are the position errors in $x$ - and $y$-direction. The velocity error caused by the wrong Doppler shift is then given as

$$
\sigma_{v_{0}}=\left|\frac{-\lambda r_{10}}{2\left(x_{0} \cos \alpha_{\mathrm{v}}+y_{0} \sin \alpha_{\mathrm{v}}\right)}\right| \sigma_{f_{\mathrm{DC}}} .
$$

For the system whose parameters are given in Table I, the velocity error is plotted in Fig. 6 for two different position errors of 5 and $20 \mathrm{~m}$. In case of $\sigma_{x_{0}}=\sigma_{y_{0}}=5 \mathrm{~m}$, the velocity error is almost below $5 \mathrm{~km} / \mathrm{h}$ for a vehicle heading between 10 and $170^{\circ}$, whereas for $\sigma_{x_{0}}=\sigma_{y_{0}}=20 \mathrm{~m}$, the error increases to $20 \mathrm{~km} / \mathrm{h}$ in the worst case.

\section{B. Utilizable Azimuth Samples}

Since no range cell migration correction is performed, only a limited number of azimuth samples around the road intersection point contain information about the moving vehicle and can be utilized for motion parameter estimation (cf. Fig. 1 right). 

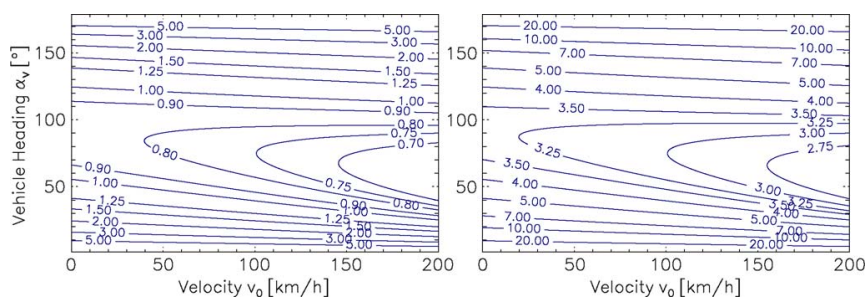

Fig. 6. Velocity estimation error in $[\mathrm{km} / \mathrm{h}]$ owing to road axis position error as a function of velocity $v_{0}$ and heading $\alpha_{\mathrm{v}}$. For computation, the system parameters given in Table I, $\theta_{i}=45^{\circ}$ and $x_{0}=0$ were used. For the left plot $\sigma_{x_{0}}=\sigma_{y_{0}}=5 \mathrm{~m}$ was assumed and for the right plot $\sigma_{x_{0}}=\sigma_{y_{0}}=20 \mathrm{~m}$.

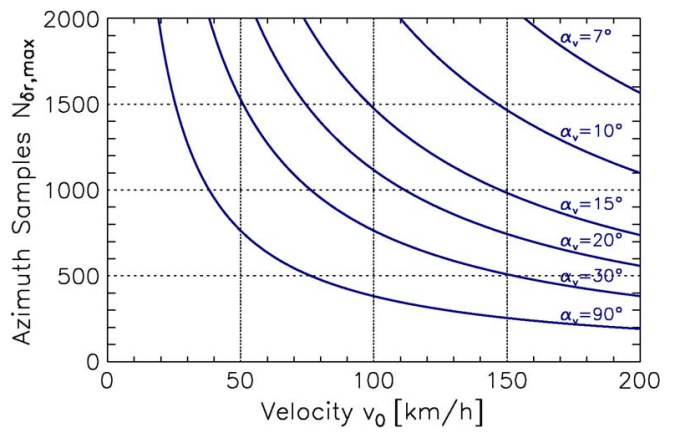

Fig. 7. Number of utilizable azimuth samples as a function of velocity $v_{0}$ and heading $\alpha_{\mathrm{v}}$ for the system whose parameters are given in Table I (incidence angle $\theta_{i}=45^{\circ}, a_{x}=a_{y}=x_{0}=0$ ).

The range sample spacing $\delta r$ as a function of range sampling frequency $f_{\mathrm{r}}$ is given as

$$
\delta r=\frac{c}{2 f_{\mathrm{r}}}
$$

The maximum number of utilizable azimuth samples (i.e., the samples which contain information about the moving vehicle) around the beam center time $t_{\mathrm{bc}}$ can be expressed as

$$
N_{\delta \mathrm{r}, \max }=2 \mathrm{PRF}\left|\Delta t_{1}\right|
$$

where $\Delta t_{1}$ is the time where the range migration corresponds to $\pm \delta r / 2$. The time $\Delta t_{1}$ in case of $f_{\mathrm{DC}} \neq 0$ can be approximated by using only the linear part of the range history given in (22)

$$
\left|\Delta t_{1}\right| \cong\left|\frac{\delta r}{\lambda f_{\mathrm{DC}}}\right|=\left|\frac{\mathrm{c}}{2 \lambda \mathrm{f}_{\mathrm{r}} f_{\mathrm{DC}}}\right| .
$$

This equation shows clearly that with decreasing range sampling frequency $f_{\mathrm{r}}$ or decreasing Doppler shift $f_{\mathrm{DC}}$ (i.e., decreasing velocity $v_{0}$ ) the time $\Delta t_{1}$ and, hence, the number of utilizable azimuth samples $N_{\delta \text { r,max }}$ increases. In Fig. 7 , it is shown how the number of azimuth samples changes with the heading of the vehicle $\alpha_{\mathrm{v}}$ and the velocity $v_{0}$. For computation, the system parameters listed in Table I were used. Even for fast moving vehicles, at least 200 azimuth samples contain information about the moving vehicle in the worst case.

The number of utilizable azimuth samples in case of $f_{\mathrm{DC}}=0$ is limited by the quadratic part of the range history in (22). The time $\Delta t_{1}$ in this case is given as

$$
\left|\Delta t_{1}\right| \cong \sqrt{\left|-\frac{2 c}{\lambda f_{\mathrm{r}} k_{\mathrm{a}}}\right|} .
$$

For the envisaged application and the system given in Table I, this time limit is less stringent than the limit given in (39) and, thus, not further considered in the paper. For instance, if a target moves antiparallel to the flight path (i.e., $\alpha_{\mathrm{v}}=180^{\circ}$ ) with a velocity of $v_{0}=180 \mathrm{~km} / \mathrm{h}$, the number of utilizable azimuth samples is $N_{\delta \text { r,max }} \cong 6900$. This value is clearly above the more stringent values shown in Fig. 7.

\section{Velocity Resolution Without Deramping}

For the following investigation, it is assumed that the "deramping" block shown in Fig. 2 is omitted in the signal processing chain. The coherent processing interval (CPI) corresponding to the number of considered azimuth samples $N_{\delta r} \leq$ $N_{\delta r, \max }$ can be written as

$$
T_{\mathrm{CPI}}=2\left|\Delta t_{1}\right|=\frac{N_{\delta \mathrm{r}}}{\mathrm{PRF}} .
$$

Considering (21), (22), and (41), the range-compressed moving vehicle signal can be modeled as

$$
\begin{array}{r}
s(t)=A\left(t-t_{\mathrm{bc}}\right) \exp \left\{-j \frac{4 \pi}{\lambda}\left[r_{10}-\frac{\lambda}{2} f_{\mathrm{DC}}\left(t-t_{\mathrm{bc}}\right)\right]\right\} \\
\times \exp \left\{j \pi k_{\mathrm{a}}\left(t-t_{\mathrm{bc}}\right)^{2}\right\} \text { rect }\left[\frac{t-t_{\mathrm{bc}}}{T_{\mathrm{CPI}}}\right]
\end{array}
$$

where the rectangular function rect[.], defined, e.g., in [21], is introduced for taking into account the limited number of azimuth samples and, hence, the limited time of the signal.

Assuming that $T_{\mathrm{CPI}}$ is much smaller than the illumination or synthetic aperture time $T_{\mathrm{SA}}$, the time variation of $A(t-$ $\left.t_{\mathrm{bc}}\right)$ can be neglected. Furthermore, if one is not interested in absolute amplitudes, the factor $A\left(t-t_{\mathrm{bc}}\right)$ can be ignored before performing a Fourier transform. The Doppler spectrum of the moving vehicle signal is then given as

$$
S\left(f_{\mathrm{a}}\right)=\int_{-\infty}^{\infty} s(t) \exp \left\{-j 2 \pi f_{\mathrm{a}} t\right\} d t
$$

where $f_{\mathrm{a}}$ is the Doppler frequency. Since (43) has no analytical solution, the "Principle of Stationary Phase" [21], [22] can be applied to get an approximation of the spectrum

$$
S\left(f_{\mathrm{a}}\right) \cong \operatorname{rect}\left[\frac{f_{\mathrm{a}}-f_{\mathrm{DC}}}{\left|k_{\mathrm{a}}\right| T_{\mathrm{CPI}}}\right] \exp \left\{j \Theta\left(f_{\mathrm{a}}\right)\right\}
$$

with

$$
\Theta\left(f_{\mathrm{a}}\right)=-\frac{4 \pi}{\lambda} r_{10}-\frac{\pi}{k_{\mathrm{a}}}\left(f_{\mathrm{a}}-f_{\mathrm{DC}}\right)^{2}-2 \pi f_{\mathrm{a}} t_{\mathrm{bc}} .
$$

From (44), it can be seen that $\left|k_{\mathrm{a}}\right| T_{\mathrm{CPI}}$ is the Doppler frequency spread or the Doppler bandwidth, respectively, of the moving vehicle signal

$$
\Delta f_{\mathrm{a}} \cong\left|k_{\mathrm{a}}\right| T_{\mathrm{CPI}}=\left|k_{\mathrm{a}}\right| \frac{N_{\delta \mathrm{r}}}{\mathrm{PRF}} .
$$

It has to be kept in mind, that the approximation in the previous equation only leads to small errors for $\left|k_{a}\right| \gg 0$. 
From the physical point of view, the minimum achievable Doppler frequency spread (without approximations) is obtained if the signal given in (42) is demodulated using a deramping function before taking the FFT [21]. After demodulation, the integral given in (43) can be solved analytically. The minimum achievable 3-dB Doppler frequency spread is then given as

$$
\Delta f_{\mathrm{a}, \min }=0.886 \frac{\mathrm{PRF}}{N_{\delta \mathrm{r}}} .
$$

Furthermore, the sample spacing in the Doppler domain is given by

$$
\delta f_{\mathrm{a}}=\frac{\mathrm{PRF}}{N_{\mathrm{FFT}}}
$$

where $N_{\mathrm{FFT}} \geq N_{\delta \mathrm{r}}$ is the number of samples contained in the signal before performing the FFT.

The achievable velocity resolution can be computed by differentiating (25) with respect to $f_{\mathrm{DC}}$ and by multiplying with the achievable frequency resolution $\Delta f_{\mathrm{a}, \text { eff }}$

$$
\Delta v_{0}=\left|\frac{-\lambda r_{10}}{2\left(x_{0} \cos \alpha_{\mathrm{v}}+y_{0} \sin \alpha_{\mathrm{v}}\right)}\right| \Delta f_{\mathrm{a}, \mathrm{eff}} .
$$

For the performance analysis regarding $\Delta v_{0}$, as achievable frequency resolution $\Delta f_{\mathrm{a} \text {,eff }}$, the maximum of the three "available" frequency resolutions is chosen, to obtain a kind of worst case estimation

$$
\Delta f_{\mathrm{a}, \mathrm{eff}}=\max \left(\Delta f_{\mathrm{a}}, \Delta f_{\mathrm{a}, \min }, \delta f_{\mathrm{a}}\right) .
$$

Using (50) with (49), the achievable velocity resolution can be computed for different system parameters, different vehicle velocities, and headings. For this analysis, additionally, an upper limit of $N_{\delta \mathrm{r}} \leq N_{\delta \mathrm{r}, \max }$ in (46) and (47) has to be considered (i.e., if $N_{\delta \mathrm{r}}$ becomes larger, $N_{\delta \mathrm{r} \text {, max }}$ has to be used in the relevant equations instead of $N_{\delta \mathrm{r}}$ ).

In Fig. 8, the achievable velocity resolutions using the system parameters given in Table I are plotted. The best performance is achieved if 256 azimuth samples are taken for processing and parameter estimation [cf. Fig. 8(b)]. In case of an incidence angle of $\theta_{i}=45^{\circ}$, the achievable velocity resolution for a vehicle heading in the range from 18 to $162^{\circ}$ is better than $5 \mathrm{~km} / \mathrm{h}$ (more or less the same results are obtained for vehicles moving in opposite road direction, i.e., for vehicle headings from 198 to $342^{\circ}$, therefore these plots are not shown). However, for steeper incidence angles, the velocity resolution becomes worse. In Fig. 8(b), for $\theta_{i}=20^{\circ}$, the achievable velocity resolution only in the vehicle heading range from 40 to $131^{\circ}$ is below $5 \mathrm{~km} / \mathrm{h}$. If a velocity resolution of $10 \mathrm{~km} / \mathrm{h}$ is sufficient enough to fulfill the requirements of the traffic monitoring application, even for $\theta_{i}=20^{\circ}$, the vehicle heading range can be extended to values between 19 and $152^{\circ}$. Outside of this vehicle heading range, the performance of the proposed GMTI algorithm is worse, and additional effort for detection and parameter estimation is necessary (cf. end of Section III-D).
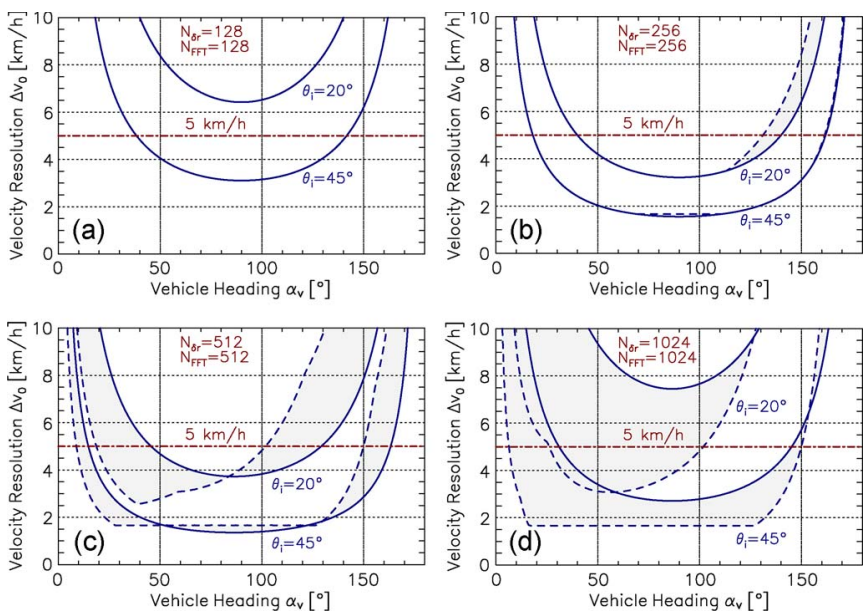

Fig. 8. Velocity resolution for two different incidence angles $\theta_{\mathrm{i}}$ and for different numbers of azimuth samples $N_{\delta \mathrm{r}}$ for the system whose parameters are given in Table I. (a) $N_{\delta \mathrm{r}}=128$, (b) $N_{\delta \mathrm{r}}=256$, (c) $N_{\delta \mathrm{r}}=512$, and (d) $N_{\delta \mathrm{r}}=1024$ (areas marked in gray: Velocity range from 10 to $180 \mathrm{~km} / \mathrm{h}$, solid blue lines: $v_{0}=10 \mathrm{~km} / \mathrm{h}$, dashed blue lines: $v_{0}=180 \mathrm{~km} / \mathrm{h}, x_{0}=a=0$ assumed for computation).

\section{Velocity Resolution Using Deramping}

The velocity resolution can be improved and the SNR increased by performing a "deramping" operation before applying the FFT (cf. Fig. 2). The "deramping" operation is based on the SPECAN algorithm [21]. For the proposed GMTI algorithm, the "deramping" function can be written as

$$
s_{\mathrm{d}}\left(t-t_{\mathrm{bc}}\right)=\exp \left\{j \pi k_{\mathrm{a}, \mathrm{st}}\left(t-t_{\mathrm{bc}}\right)^{2}\right\}
$$

where $k_{\mathrm{a}, \mathrm{st}}$ is the Doppler slope of a stationary target located at the observed road point. The stationary Doppler slope is obtained from (24) by setting all motion parameters of the vehicle to zero

$$
k_{\mathrm{a}, \mathrm{st}}=-\frac{2 v_{\mathrm{p}}^{2}}{\lambda r_{10}}\left(1-\frac{x_{0}^{2}}{r_{10}^{2}}\right) .
$$

The deramping operation is a simple multiplication in time domain $I(t)=s(t) s_{\mathrm{d}}^{*}\left(t-t_{\mathrm{bc}}\right)$. The obtained deramped moving vehicle signal can then be written as

$$
\begin{gathered}
I(t)=A\left(t-t_{\mathrm{bc}}\right) \exp \left\{-j \frac{4 \pi}{\lambda}\left[r_{10}-\frac{\lambda}{2} f_{\mathrm{DC}}\left(t-t_{\mathrm{bc}}\right)\right]\right\} \\
\times \exp \left\{j \pi \Delta k_{\mathrm{a}}\left(t-t_{\mathrm{bc}}\right)^{2}\right\} \operatorname{rect}\left[\frac{t-t_{\mathrm{bc}}}{T_{\mathrm{CPI}}}\right]
\end{gathered}
$$

with

$$
\Delta k_{\mathrm{a}}=k_{\mathrm{a}}-k_{\mathrm{a}, \mathrm{st}} .
$$

Note that not the total ramp of each moving vehicle signal is removed, but only the part of the ramp caused by the motion of the platform carrying the radar. A residual ramp $\Delta k_{\mathrm{a}}$ remains. After deramping, the Doppler shift $f_{\mathrm{DC}}$ of the moving vehicle signal is not changed since for deramping the known beamcenter time $t_{\mathrm{bc}}$ is used. 

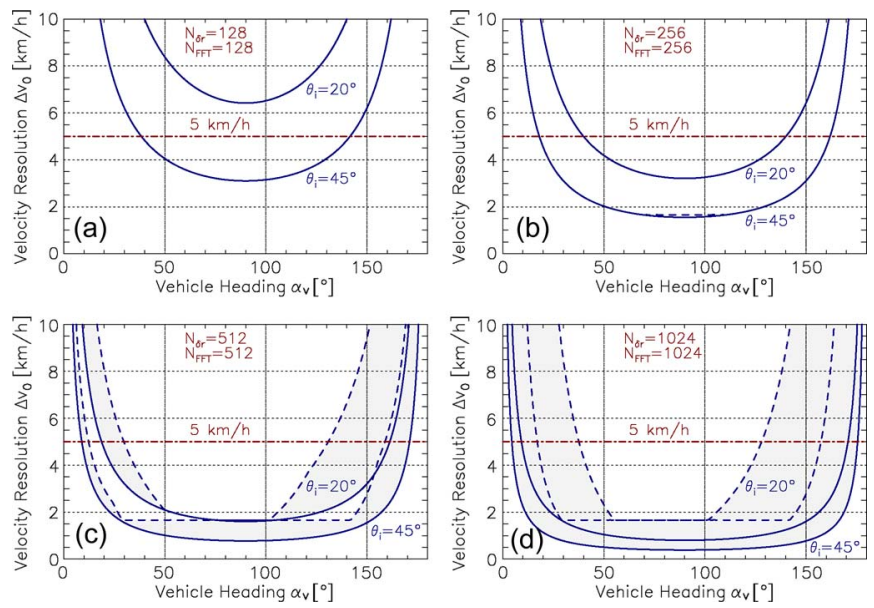

Fig. 9. Velocity resolution after "deramping" for two different incidence angles $\theta_{\mathrm{i}}$ and for different numbers of azimuth samples $N_{\delta \mathrm{r}}$ for the system whose parameters are given in Table I. (a) $N_{\delta \mathrm{r}}=128$, (b) $N_{\delta \mathrm{r}}=256$, (c) $N_{\delta \mathrm{r}}=512$ and (d) $N_{\delta \mathrm{r}}=1024$ (areas marked in gray: velocity range from 10 to $180 \mathrm{~km} / \mathrm{h}$, solid blue lines: $v_{0}=10 \mathrm{~km} / \mathrm{h}$, dashed blue lines: $v_{0}=180 \mathrm{~km} / \mathrm{h}, x_{0}=a=0$ assumed for computation).

The Doppler frequency spread of the moving vehicle signal, after deramping and application of the FFT, can be approximated as (same derivation as in Section III-C)

$$
\Delta f_{\mathrm{a}, \mathrm{d}} \cong\left|\Delta k_{\mathrm{a}}\right| T_{\mathrm{CPI}}=\left|\Delta k_{\mathrm{a}}\right| \frac{N_{\delta \mathrm{r}}}{\mathrm{PRF}} .
$$

Again, the above approximation only holds if $N_{\delta \mathrm{r}} \leq N_{\delta \mathrm{r}, \max }$ and $\|\Delta k\| \gg 0$. In case of $\Delta k_{\mathrm{a}}=0$, the exact 3-dB frequency spread is given in (47).

In Fig. 9, the achievable velocity resolutions after "deramping" for the system given in Table I are shown. Again, (49) and (50) were applied, but now with $\Delta f_{\mathrm{a}, \mathrm{d}}$ instead of $\Delta f_{\mathrm{a}}$. As in the previous subsection, where no "deramping" was performed, the best performance is achieved if 256 azimuth samples are used [cf. Fig. 9(b)]. By comparing Fig. 9(b) with Fig. 8(b) it can be seen that the achievable velocity resolution is quite similar, apart from the vehicle heading region from 115 to $160^{\circ}$, where the velocity resolution without "deramping" is maximal $2 \mathrm{~km} / \mathrm{h}$ worse than with "deramping." As a consequence, for very time critical applications, the "deramping" operation can be omitted by introducing only an insignificantly larger velocity resolution in a limited vehicle heading region.

As can be seen in the plots in Figs. 8 and 9, the performance (i.e., the achievable velocity resolution) of the proposed GMTI algorithm becomes worse for vehicles moving nearly parallel or antiparallel to the flight path. As mentioned before, for these small vehicle headings, additional effort for detection and parameter estimation is necessary.

\section{E. Velocity Resolution Using Adaptive Deramping}

For small vehicle headings, the range cell migration becomes smaller and, thus, the number of utilizable azimuth samples increases (cf. Fig. 7). The velocity resolution can be kept low for a larger vehicle heading range if "Adaptive deramping" is used. "Adaptive deramping" is similar to the matched-filter bank approach, and therefore it requires more computational
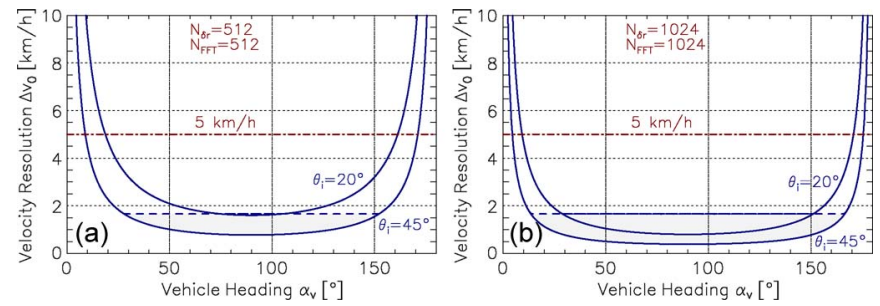

Fig. 10. Velocity resolution after "adaptive deramping" for two different incidence angles $\theta_{\mathrm{i}}$ and for different numbers of azimuth samples $N_{\delta \mathrm{r}}$ for the system whose parameters are given in Table I. (a) $N_{\delta \mathrm{r}}=512$, (b) $N_{\delta \mathrm{r}}=1024$ (areas marked in gray: velocity range from 10 to $180 \mathrm{~km} / \mathrm{h}$, solid blue lines: $v_{0}=10 \mathrm{~km} / \mathrm{h}$, dashed blue lines: $v_{0}=180 \mathrm{~km} / \mathrm{h}, x_{0}=a=0$ assumed for computation).

load. By "adaptive deramping," the moving vehicle signal is successively deramped using different assumptions of the Doppler slope $\hat{k}_{\mathrm{a}}$. The Doppler slope $\hat{k}_{\mathrm{a}}$, which leads after "deramping" and FFT to the maximum peak in Doppler domain, is most likely the true Doppler slope of the moving vehicle signal. The "adaptive deramping" function can be expressed as

$$
\hat{s}_{\mathrm{d}}\left(t-t_{\mathrm{bc}}\right)=\exp \left\{j \pi \hat{k}_{\mathrm{a}}\left(t-t_{\mathrm{bc}}\right)^{2}\right\} .
$$

Under the assumption that by "adaptive deramping," the exact Doppler slope $k_{\mathrm{a}}$ can be estimated, the velocity resolution shown in Fig. 10 for the system in Table I can be achieved. Comparing Fig. 10 to Figs. 8 and 9, now the velocity resolution is only worse than $5 \mathrm{~km} / \mathrm{h}$ in the vehicle heading range from $0^{\circ}$ to $10^{\circ}$ and from $170^{\circ}$ to $180^{\circ}$.

Remember that the road angle $\alpha$ as well as the incidence angle $\theta_{\mathrm{i}}$ are known a priori. Thus, a decision if "deramping" or "adaptive deramping" should be used for parameter estimation or not, can be made automatically by considering the desired velocity resolution $\Delta v_{0}$. For the system in Table I, for example, the following simple decisions regarding the "right" estimation procedure can be made, if a velocity resolution smaller than $5 \mathrm{~km} / \mathrm{h}$ should be achieved for nearly all possible vehicle heading angles:

- For road angles $40^{\circ} \leq|\alpha| \leq 140^{\circ}$, only $N_{\delta \mathrm{r}}=256$ azimuth samples are extracted, and a "deramping" using the stationary Doppler slope $k_{\mathrm{a}, \mathrm{st}}$ is performed before taking the FFT and before performing motion parameter estimation.

- For road angles $10^{\circ} \leq|\alpha|<40^{\circ}$ and $140^{\circ} \leq|\alpha| \leq 170^{\circ}$ for each road point $N_{\delta \mathrm{r}}=1024$, azimuth samples are extracted, and "adaptive deramping" should be performed.

For road angles $0^{\circ} \leq|\alpha|<10^{\circ}$ and $170^{\circ}<|\alpha|<180^{\circ}$, neither "deramping" nor "adaptive deramping" leads to the desired velocity resolution. Thus, for roads lying nearly parallel or antiparallel to the flight direction, the performance of the proposed a priori knowledge-based GMTI approach suffers. A short discussion about such roads is given in the next subsection.

\section{F. Roads Parallel to Flight Direction}

For vehicles moving nearly parallel or antiparallel to the flight path, no distinct intersection of the road axis with the range-compressed moving vehicle signal exists. As a 
consequence, one and the same vehicle can cause several detections. On the other hand, a larger amount of azimuth samples can be exploited for parameter estimation. For the system in Table I and for a vehicle heading of $\alpha_{\mathrm{v}}=10^{\circ}$, at least 1100 azimuth samples are usable (cf. Fig. 7), for $\alpha_{\mathrm{v}}=5^{\circ}$ about 2200 samples, and for $\alpha_{\mathrm{v}}=0^{\circ}$ even 6000. These numbers of samples correspond to observation times of $0.22,0.44$, and $1.2 \mathrm{~s}$. The observation times can further be increased by reducing the range sampling frequency $f_{\mathrm{r}}$ or by taking into account the range migration. Thus, for roads lying nearly parallel or antiparallel to the flight path, it is suggested to use the conventional matched-filter bank approach [5], [16] in combination with along-track interferometry (ATI) and a powerful clustering algorithm. In this case, velocity and position estimation are performed by exploiting the estimated ATI phase and the Doppler slope $\hat{k}_{\mathrm{a}}$, but not direcly the estimated Doppler shift $f_{\mathrm{DC}}$. However, for an optimum incorporation of the matchedfilter bank and ATI into the proposed a priori knowledgebased GMTI processing framework, additional comprehensive investigations are necessary. We leave these investigations as an open topic for the future.

\section{G. Detectable Velocity Range}

Having a single-channel system without clutter suppression capability, only moving vehicle signals with Doppler shifts lying outside the clutter band can be detected [23]. The clutter bandwidth is given as [21]

$$
B_{\mathrm{c}}=0.886 \frac{2 v_{\mathrm{p}} \cos \psi}{L_{\mathrm{a}}}
$$

where $L_{\mathrm{a}}$ is the antenna length in azimuth. For computing the minimum detectable velocity $v_{0, \min }(\mathrm{MDV})$, for a singlechannel system, the condition $\left|f_{\mathrm{DC}}\right| \geq\left|f_{\mathrm{DC}, \mathrm{st}}\right|+B_{c} / 2$ has to be fulfilled. The MDV is then given as

$$
v_{0, \min } \geq\left|\frac{\lambda r_{10}}{x_{0} \cos \alpha_{\mathrm{v}}+y_{0} \sin \alpha_{\mathrm{v}}} \cdot \frac{0.443 v_{\mathrm{p}} \cos \psi}{L_{\mathrm{a}}}\right| .
$$

The MDV can be significantly decreased by using dual- and multichannel systems and applying sophisticated clutter suppression techniques like DPCA or STAP, respectively. Detailed theoretical analyses can be found in [7], [19], [20] and should not be repeated here.

For avoiding Doppler ambiguities, the limit for the maximum unambiguously detectable velocity $v_{0, \max }$ is determined by the PRF. Using the condition $\left|f_{\mathrm{DC}}\right| \leq\left|f_{\mathrm{DC}, \mathrm{st}}\right|+\mathrm{PRF} / 2$, the velocity $v_{0, \max }$ can be expressed as

$$
v_{0, \max } \leq\left|\frac{\lambda r_{10}}{x_{0} \cos \alpha_{\mathrm{v}}+y_{0} \sin \alpha_{\mathrm{v}}} \cdot \frac{\mathrm{PRF}}{4}\right| .
$$

The maximum detectable velocity $v_{0, \max }$ in the classical way only can be increased by increasing the PRF. However, Doppler ambiguities can be resolved by taking into account the range migration of the moving vehicle signal as shown in [9], [24], without the need of increasing the PRF. For the proposed algorithm, the linear range migration and, hence, the unambiguous Doppler shift can be estimated from the clutter suppressed data

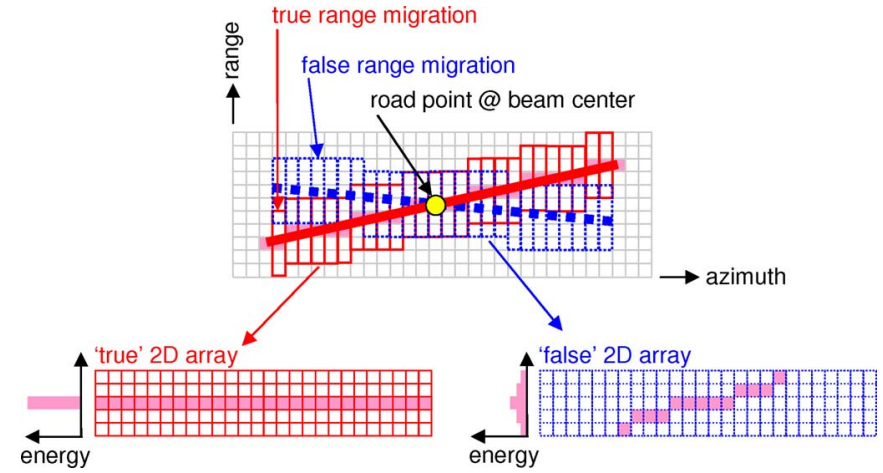

Fig. 11. Principle of linear range migration estimation and Doppler ambiguity resolution using a "Quick and Dirty Radon Transformation."

without the need of expensive 2-D matched filtering. From (22), the linear range migration can be expressed as

$$
\Delta r(t)=-\frac{\lambda}{2} f_{\mathrm{DC}}\left(t-t_{\mathrm{bc}}\right)=k_{\Delta \mathrm{r}}\left(t-t_{\mathrm{bc}}\right)
$$

where $k_{\Delta \mathrm{r}}$ is the slope of the linear range migration. In case of Doppler backfolding, the estimated ambiguous Doppler shift $\hat{f}_{\mathrm{DC}}$ is related to the "true" unambiguous Doppler shift in the following way:

$$
f_{\mathrm{DC}}=\hat{f}_{\mathrm{DC}}+n \cdot \mathrm{PRF}
$$

where $n$ is an integer number. The maximum value of $n$ is determined by $n_{\max }=f_{\mathrm{DC}, \max } / \mathrm{PRF}$ and known a priori ( $f_{\mathrm{DC}, \max }$ can be computed with $(23)$ by considering the expected maximum velocity of a moving vehicle). If one can estimate the linear range migration slope $k_{\Delta \mathrm{r}}$, the integer $n$ and, hence, the "true" unambiguous Doppler shift can be computed

$$
n=\operatorname{round}\left[-\frac{1}{\mathrm{PRF}}\left(\frac{2 k_{\Delta \mathrm{r}}}{\lambda}+\hat{f}_{\mathrm{DC}}\right)\right] \text {. }
$$

The range migration slope $k_{\Delta \mathrm{r}}$ can be estimated by using for example the Radon transformation [25] or the Hough transformation [26], respectively. However, in practice, the range migration slope can only adopt discrete values given by

$$
k_{\Delta \mathrm{r}}=-\frac{\lambda}{2}\left(\hat{f}_{\mathrm{DC}}+n \cdot \mathrm{PRF}\right)
$$

so that only a few discrete range migration slopes (i.e., a total number of $\left(2 \cdot n_{\max }+1\right)$ slopes $)$ have to be evaluated. This can be done by using a kind of "Quick and Dirty Radon Transformation" (QDRT). Here, for each assumed range migration slope, a different 2-D array is extracted around the beam center position (cf. Fig. 11). The extracted 2-D array has to have a larger number of azimuth samples (larger than the number needed for vehicle detection and ambiguous Doppler shift $\hat{f}_{\mathrm{DC}}$ estimation), so that a range walk through several range bins is observable. For the "true" range migration slope, the whole signal energy will be more or less concentrated along a horizontal line in the 2-D array (cf. Fig. 11 bottom left). By comparing the total energy in the horizontal lines of all generated 2-D arrays with each other, the 2-D array corresponding to the correct range migration slope and, thus, corresponding to the "true" 


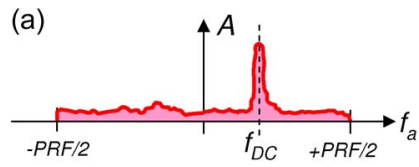

(c)
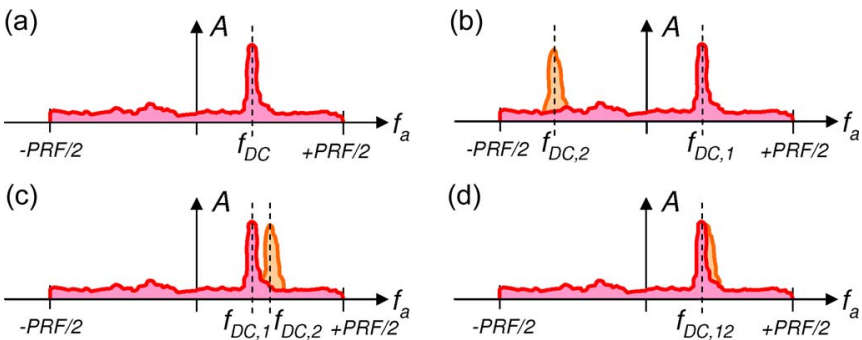

(d)

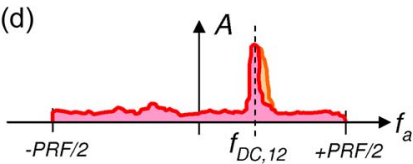

Fig. 12. Exemplary Doppler spectrum of a single vehicle (a), two vehicles moving in opposite directions (b), two vehicles moving in same direction with a certain velocity difference (c), and two vehicles moving with the same velocity in the same direction at the same range (d).
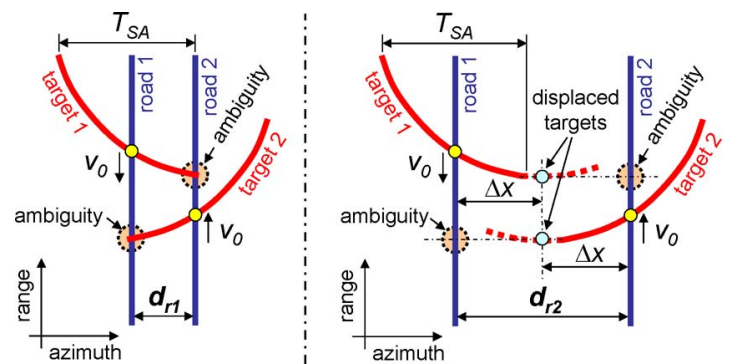

Fig. 13. Minimum road distances $d_{\mathrm{r} 1}$ and $d_{\mathrm{r} 2}$ for avoiding false detections and ambiguities (left: proposed algorithm; right: displacement-based algorithm).

Doppler shift can be found (the correct 2-D array contains the maximum energy in one of its horizontal lines).

The QDRT approach was verified using experimental data (cf. Section IV-B).

\section{H. Multicomponent Signals}

Independent of the number of road lanes, for the proposed algorithm only the center road axis is mapped into the SAR data array but not each lane. Having several lanes, under circumstances, it may happen that several vehicles on the road move at the same range. As a consequence, their range histories overlap each other. Thus, in the azimuth samples taken for parameter estimation, the signals of several moving vehicles are included. Additional effort is necessary to separate these signals. In the simplest cases, where the vehicles move with different velocities or in different directions [cf. Fig. 12(a)-(c)], a separation directly in the Doppler domain is feasible if the velocity difference is larger than the velocity resolution given in (49).

The most complex case occurs, if the vehicles at the same range move into the same direction with nearly the same velocities so that the signals have the same Doppler shift [cf. Fig. 12(d)]. In this particular case, a vehicle separation in the Doppler domain is impossible.

\section{Ambiguities and False Detections}

For the proposed algorithm, false detections and ambiguities have principally two different sources: unsuppressed stationary target signals (residual clutter) and signals from vehicles moving on adjacent roads. Signals from vehicles moving on adjacent roads may lead to false detections and ambiguities or wrong road assignments, respectively (cf. Fig. 13 left). As

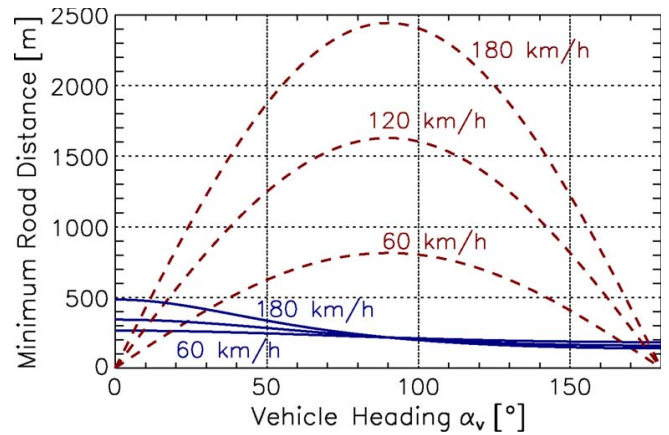

Fig. 14. Minimum road distances for avoiding false detections and ambiguities as a function of vehicle heading and velocity for the specific system whose parameters are listed in Table I $\left(\theta_{i}=45^{\circ}, x_{0}=\psi=0\right.$ assumed; blue solid: proposed algorithm, $d_{\mathrm{r} 1}$; red dashed: displacement-based algorithm, $d_{\mathrm{r} 2}$ ).

a result of a wrong road assignment, the expected position of the target as well as the estimates of the motion parameters are wrong. For the proposed algorithm, the minimum distance in along-track direction between adjacent roads for avoiding wrong road assignments is mainly determined by the $3-\mathrm{dB}$ beamwidth of the azimuth antenna pattern and the relative along-track velocity between the radar and the vehicle. The maximum observation time of the vehicle signal determined by the azimuth antenna pattern (here range migration does not matter) can be approximated as

$$
T_{\mathrm{SA}} \cong 0.886 \frac{\lambda r_{10}}{L_{\mathrm{a}}\left(v_{\mathrm{p}}-v_{0} \cos \alpha_{\mathrm{v}}\right) \cos \psi} .
$$

The minimum distance between adjacent roads can then be expressed as (cf. Fig. 13 left)

$$
d_{\mathrm{r} 1}=\frac{T_{\mathrm{SA}}}{2} v_{\mathrm{p}}=0.443 \frac{\lambda r_{10}}{L_{\mathrm{a}} \cos \psi}\left(\frac{v_{\mathrm{p}}}{v_{\mathrm{p}}-v_{0} \cos \alpha_{\mathrm{v}}}\right) .
$$

In contrast to the proposed algorithm, the minimum road distance using displacement-based algorithms is much larger [8]. The azimuth displacement after SAR azimuth focusing using the full bandwidth determined by the PRF can be approximated as (it is assumed that the PRF is high enough so that no Doppler backfolding of the signal occurs)

$$
\Delta x \cong-\frac{f_{\mathrm{DC}}-f_{\mathrm{DC}, \mathrm{st}}}{k_{\mathrm{a}, \mathrm{st}}} v_{\mathrm{p}}=-\frac{v_{0}}{v_{\mathrm{p}}}\left(x_{0} \cos \alpha_{\mathrm{v}}+y_{0} \sin \alpha_{\mathrm{v}}\right)
$$

and the minimum distance between adjacent roads for avoiding ambiguities is then (cf. Fig. 13 right)

$$
d_{\mathrm{r} 2}=2|\Delta x| \text {. }
$$

For the comparison of the road distances shown in Fig. 14, the system parameters listed in Table I were used, and a nonsquinted case (i.e., $x_{0}=0$ ) was assumed. It can be seen that the roads can be closer together if the proposed GMTI algorithm is used.

False detections or ambiguities, respectively, in general cannot be resolved with a single-channel system. Having a multichannel system, the direction-of-arrival (DOA) angle can be estimated additionally. If the DOA angle is not the same as the squint angle $\psi$, the detection is a false alarm or an ambiguity 
and can be discarded. Hence, using multichannel systems, the minimum road distance can be significantly decreased. The minimum road distance is then limited by the standard deviation of the DOA estimator [7], [27].

For a dual-channel system, the ATI phase of the coregistered and Fourier transformed signals, received by the fore and the aft channel, can be approximated as (using again the "Principle of Stationary Phase")

$$
\begin{array}{r}
\Phi_{\text {ATI }}\left(f_{\mathrm{a}}\right)=\frac{2 \pi}{\lambda} d_{\mathrm{a}} \frac{x_{0}}{r_{10}}+2 \pi d_{\mathrm{a}} \frac{v_{0} \cos \alpha_{\mathrm{v}}-v_{\mathrm{p}}}{\lambda k_{\mathrm{a}} r_{10}}\left(f_{\mathrm{a}}-f_{\mathrm{DC}}\right) \\
+\pi k_{\mathrm{a}}\left(d_{\mathrm{a}} \frac{v_{0} \cos \alpha_{\mathrm{v}}-v_{\mathrm{p}}}{\lambda k_{\mathrm{a}} r_{10}}\right)^{2}-2 \pi f_{\mathrm{a}} \frac{d_{\mathrm{a}}}{2 v_{\mathrm{p}}}
\end{array}
$$

where $d_{\mathrm{a}}$ is the physical separation of the receiving antennas in azimuth direction. The ATI phase at the peak position $f_{\mathrm{a}}=f_{\mathrm{DC}}$ is then given as

$\Phi_{\mathrm{ATI}, \mathrm{f}_{\mathrm{DC}}}=\frac{2 \pi}{\lambda} d_{\mathrm{a}} \frac{x_{0}}{r_{10}}+\pi \frac{d_{\mathrm{a}}^{2}}{\lambda^{2}} \frac{\left(v_{0} \cos \alpha_{\mathrm{v}}-v_{\mathrm{p}}\right)^{2}}{k_{\mathrm{a}} r_{10}^{2}}-\pi f_{\mathrm{DC}} \frac{d_{\mathrm{a}}}{v_{\mathrm{p}}}$.

The second term in the previous equation is negligibly small compared to the other terms and can therefore be neglected. Substituting $x_{0} / r_{10}=\sin \psi_{\mathrm{DOA}}$ in the previous equation, the DOA angle can be computed

$$
\psi_{\mathrm{DOA}}=\arcsin \left(\frac{\lambda}{2 \pi d_{\mathrm{a}}} \Phi_{\mathrm{ATI}, \mathrm{f}_{\mathrm{DC}}}+\frac{\lambda}{2 v_{\mathrm{p}}} f_{\mathrm{DC}}\right) .
$$

However, since in practice, $\Phi_{\mathrm{ATI}, \mathrm{f}_{\mathrm{DC}}}$ only can be measured in fractions of $2 \pi$, above equation has to be modified to

$\psi_{\mathrm{DOA}, \mathrm{m}}=\arcsin \left[\frac{\lambda}{2 \pi d_{\mathrm{a}}}\left(\Phi_{\mathrm{ATI}, \mathrm{f}_{\mathrm{DC}}}+m \cdot 2 \pi\right)+\frac{\lambda}{2 v_{\mathrm{p}}} f_{\mathrm{DC}}\right]$

where $m$ is an integer.

By comparing all estimated DOA angles $\psi_{\mathrm{DOA}, \mathrm{m}}$ with the squint angle $\psi$, false detections can be discarded to a certain degree. Nevertheless, particularly in the dual-channel case, the ATI phase is contaminated by clutter so that the performance of the DOA estimation suffers.

\section{EXPERIMENTAL DATA}

In 2007, several GMTI experiments have been performed using DLR's new multichannel and multifrequency F-SAR system [28]. As test sites, the former military airfield in Memmingen and a region around Chiemsee, both located in Germany, have been used. F-SAR was operated in X-band with a range bandwidth of $100 \mathrm{MHz}$ in a dual-channel mode with an effective channel PRF of $2.5 \mathrm{kHz}$ [29]. The effective along-track baseline between the receiving antennas was $10 \mathrm{~cm}$. The aircraft flew each time at an altitude of approximately $2200 \mathrm{~m}$ above ground, so that the typical incidence angle range from $25^{\circ}$ to $60^{\circ}$ corresponds to slant ranges in the order of 2400 to $4400 \mathrm{~m}$.

For the Memmingen test site, conventional passenger cars were used as controlled moving vehicles. Some of them were equipped with radar reflectors to enhance the RCS, as well as with differential GPS (DGPS) receivers to retrieve reliable geographical reference positions and velocities for a sophisticated GMTI algorithm verification. Additionally, simultaneous with
TABLE II

Dual-Channel Results: Across-Track Motion

\begin{tabular}{cccccc}
\hline $\begin{array}{c}v_{0, \text { optical }} \\
{[\mathrm{km} / \mathrm{h}]}\end{array}$ & $\begin{array}{c}v_{0, \mathrm{DGPS}} \\
{[\mathrm{km} / \mathrm{h}]}\end{array}$ & $\begin{array}{c}\hat{v}_{0} \\
{[\mathrm{~km} / \mathrm{h}]}\end{array}$ & $\begin{array}{c}\Delta v_{0} \\
{[\mathrm{~km} / \mathrm{h}]}\end{array}$ & $\begin{array}{c}\text { Abs. Position Error } \\
{[\mathrm{m}]}\end{array}$ & $\begin{array}{c}r_{10} \\
{[\mathrm{~m}]}\end{array}$ \\
\hline 7.1 & $\mathrm{n} / \mathrm{a}$ & 8.6 & 1.5 & 17.9 & 2881.5 \\
80.8 & $\mathrm{n} / \mathrm{a}$ & 84.3 & 3.5 & 9.9 & 2901.0 \\
16.0 & $\mathrm{n} / \mathrm{a}$ & 14.2 & -1.8 & 17.3 & 2941.6 \\
44.0 & 44.0 & 42.7 & -1.3 & 16.5 & 3081.6 \\
\hline
\end{tabular}

the radar, also optical images from the same scene were taken to retrieve also knowledge about other road vehicles.

In the Chiemsee region, vehicles of opportunity (passenger cars and trucks) were monitored on the autobahn A8. Unfortunately, no reliable ground truth data were available for these vehicles. Nevertheless, the estimated velocities between 79 and $149 \mathrm{~km} / \mathrm{h}$ seem reasonable.

For the practical implementation of the algorithm (cf. Fig. 2), the freely available OpenStreetMap [30] is used as road database. The elevations corresponding to the road points (OpenStreetMap contains no elevation information) are obtained from the free Shuttle Radar Topography Mission (SRTM) digital elevation model [31]. For obtaining the experimental results shown in the following subsections, the dualchannel algorithm structure shown in Fig. 2 was used. Since we are mainly interested in showing the parameter estimation, but not the detection performance, the DPCA detection threshold was fixed to a certain SCNR value. Also, the DOA angle difference for skipping false detections was set to a fixed threshold.

\section{A. Over-Sampled Data in Azimuth}

In Fig. 15, some GMTI results obtained from a dual-channel data take acquired over the Memmingen airfield are shown. The used PRF of $2500 \mathrm{~Hz}$ was high enough for avoiding Doppler ambiguities. During the data take all controlled vehicles have moved in across-track direction. The average clutter Doppler centroid was $186 \mathrm{~Hz}$, corresponding to a squint angle of approximately $1.8^{\circ}$. The estimated velocities $\hat{v}_{0}$ of the vehicles are (cf. Table II): 8.6, 84.3, 14.2, and $42.7 \mathrm{~km} / \mathrm{h}$. Compared to the optical reference data, the velocity estimation errors $\Delta v_{0}$ are: $-1.5,3.5,-1.8$, and $-1.3 \mathrm{~km} / \mathrm{h}$. The corresponding absolute position errors are: $17.9,9.9,17.3$, and $16.5 \mathrm{~m}$. The runway in Memmingen is about $30 \mathrm{~m}$ broad, and as road axis for the coordinate transformation, the middle of the runway was chosen, but during the experiment, the vehicles have moved along the edge. This fact explains a position estimation error in the order of $15 \mathrm{~m}$. Furthermore, the accuracy of the optical reference data itself is also limited to about $\pm 3.5 \mathrm{~km} / \mathrm{h}$ velocity accuracy and to \pm 5 to $\pm 15 \mathrm{~m}$ absolute position accuracy. Under this aspect, the obtained accuracy of the GMTI processor is quite good.

Additionally, the velocity estimate of the vehicle moving with $42.7 \mathrm{~km} / \mathrm{h}$ was verified using the GPS reference data as shown in Fig. 16. The GPS velocity corresponding to the estimated beam center time $t_{\mathrm{bc}}(13: 33: 37.0$ UTC) is $44 \mathrm{~km} / \mathrm{h}$. The velocity estimation error is $-1.3 \mathrm{~km} / \mathrm{h}$, which is the same as using the optical reference data. 

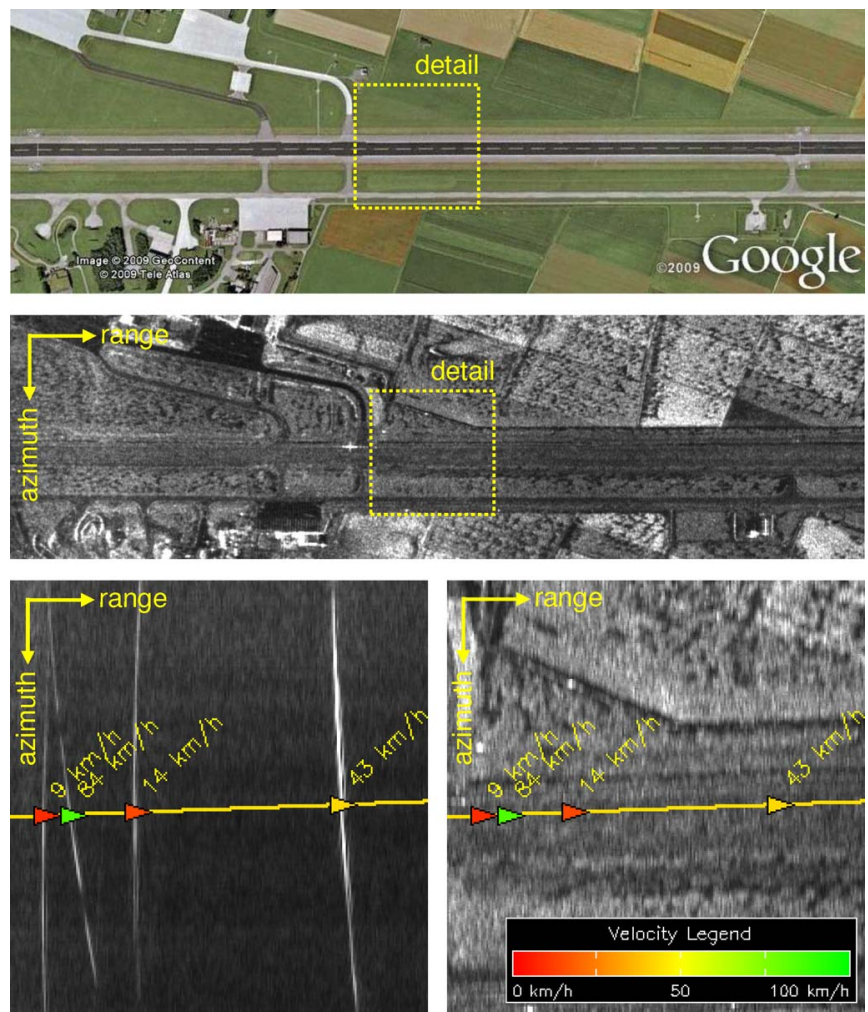

Fig. 15. Optical Google Earth image (top) and SAR image (middle, data take rc07trmrad0101x1) of Memmingen airfield, range-compressed DPCA image of the "detail" with overlaid runway axis and detected moving vehicles as triangles (bottom left), and corresponding SAR image (bottom right).
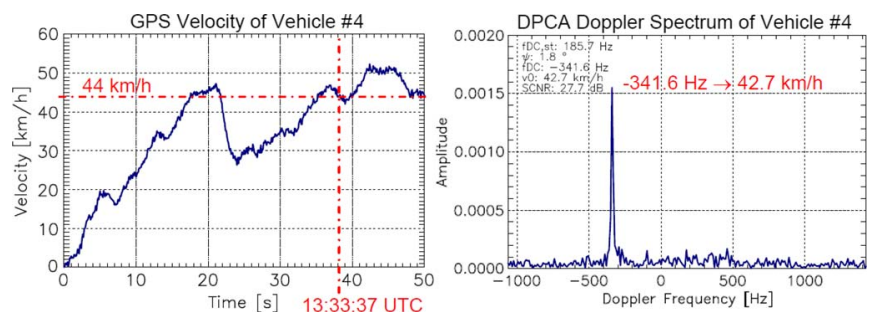

Fig. 16. Verification of the estimated beam center velocity of the vehicle no 4 (estimated velocity of $42.7 \mathrm{~km} / \mathrm{h}$ ) using DGPS data as reference (left: DGPS velocity of the vehicle, right: DPCA Doppler spectrum of the vehicle signal).
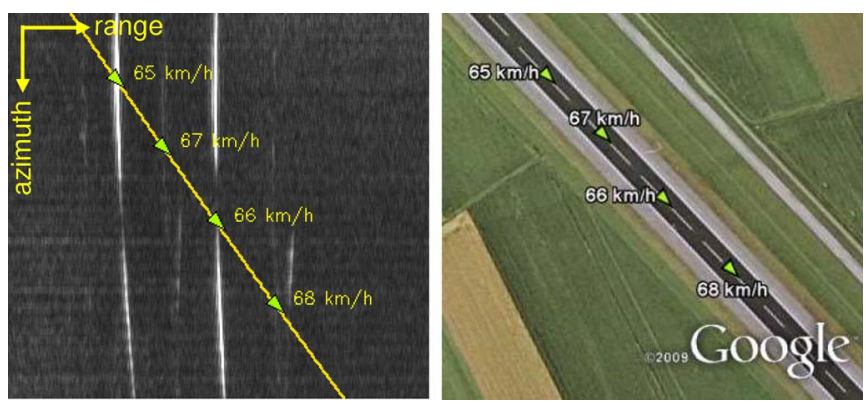

Fig. 17. Range-compressed DPCA image of data take rc07trmrad0103x 1 (left) and corresponding Google Earth image (right), both with overlaid moving vehicle symbols. The two vehicles appearing very bright in the DPCA image were equipped with special radar reflectors to enhance the RCS.

For smaller road angles $\alpha$, the performance of the algorithm decreases. In Fig. 17, the GMTI results for the runway at an angle of $\alpha=45^{\circ}$ are shown. The average Doppler was

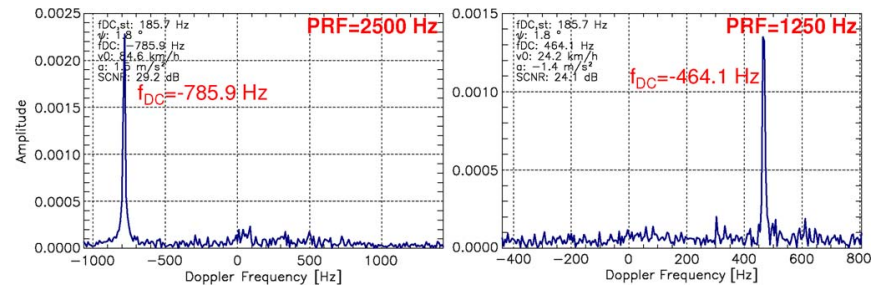

Fig. 18. DPCA Doppler spectrum of the vehicle moving with about $84 \mathrm{~km} / \mathrm{h}$ in case of over-sampling with PRF $=2500 \mathrm{~Hz}$ (left) and under-sampling with $\mathrm{PRF}=1250 \mathrm{~Hz}$ (right).
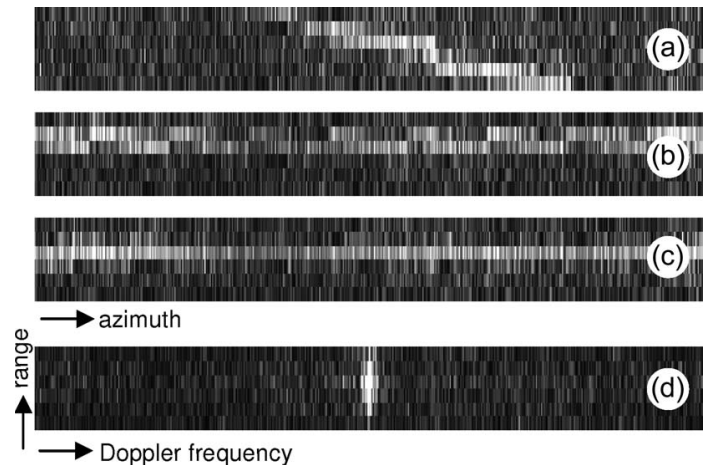

Fig. 19. Undersampled DPCA range/azimuth arrays (1024 azimuth $\times 6$ range samples) taken around the road point where the vehicle moves at beam center time. (a) Wrong assumption of the Doppler shift. (b) Correct Doppler shift assumption. (c) Moving vehicle signal aligned along range. (d) Range/Doppler image of focused moving vehicle signal after application of "deramping" with the estimated Doppler slope).
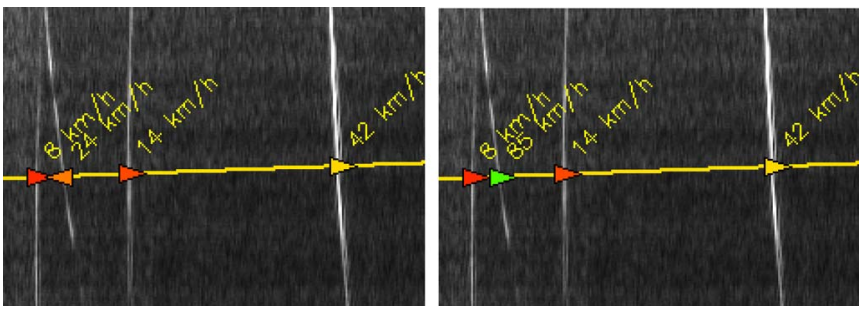

Fig. 20. GMTI results without Doppler ambiguity resolving (left) and after applying the proposed technique (right). In the first case, the vehicle moves with $24.2 \mathrm{~km} / \mathrm{h}$ into the wrong direction (absolute velocity error of $56.6 \mathrm{~km} / \mathrm{h}$ ), and in the second case, the estimated vehicle velocity is $84.6 \mathrm{~km} / \mathrm{h}$ (error of only $3.8 \mathrm{~km} / \mathrm{h}$, right direction).

abnormally large at $491 \mathrm{~Hz}$ ( $4.8^{\circ}$ squint angle). Compared to the optical reference data, the largest velocity error is $9.3 \mathrm{~km} / \mathrm{h}$, and the largest position error $26.4 \mathrm{~m}$. During data acquisition, the weather was bad and windy, and so the residual motion compensation error might influence the GMTI processor performance. However, we think that an error below $10 \mathrm{~km} / \mathrm{h}$ is still good enough for many traffic monitoring applications.

In the "formatting" stage of the automatic GMTI processing chain also Keyhole Markup Language files are produced, which easily can be visualized using Google Earth [32], as shown in Fig. 17, right.

\section{B. Under-Sampled Data in Azimuth}

As already mentioned in the previous sections, Doppler ambiguities occurring due to fast moving vehicles in combination with a low PRF can be resolved by taking into account the range 


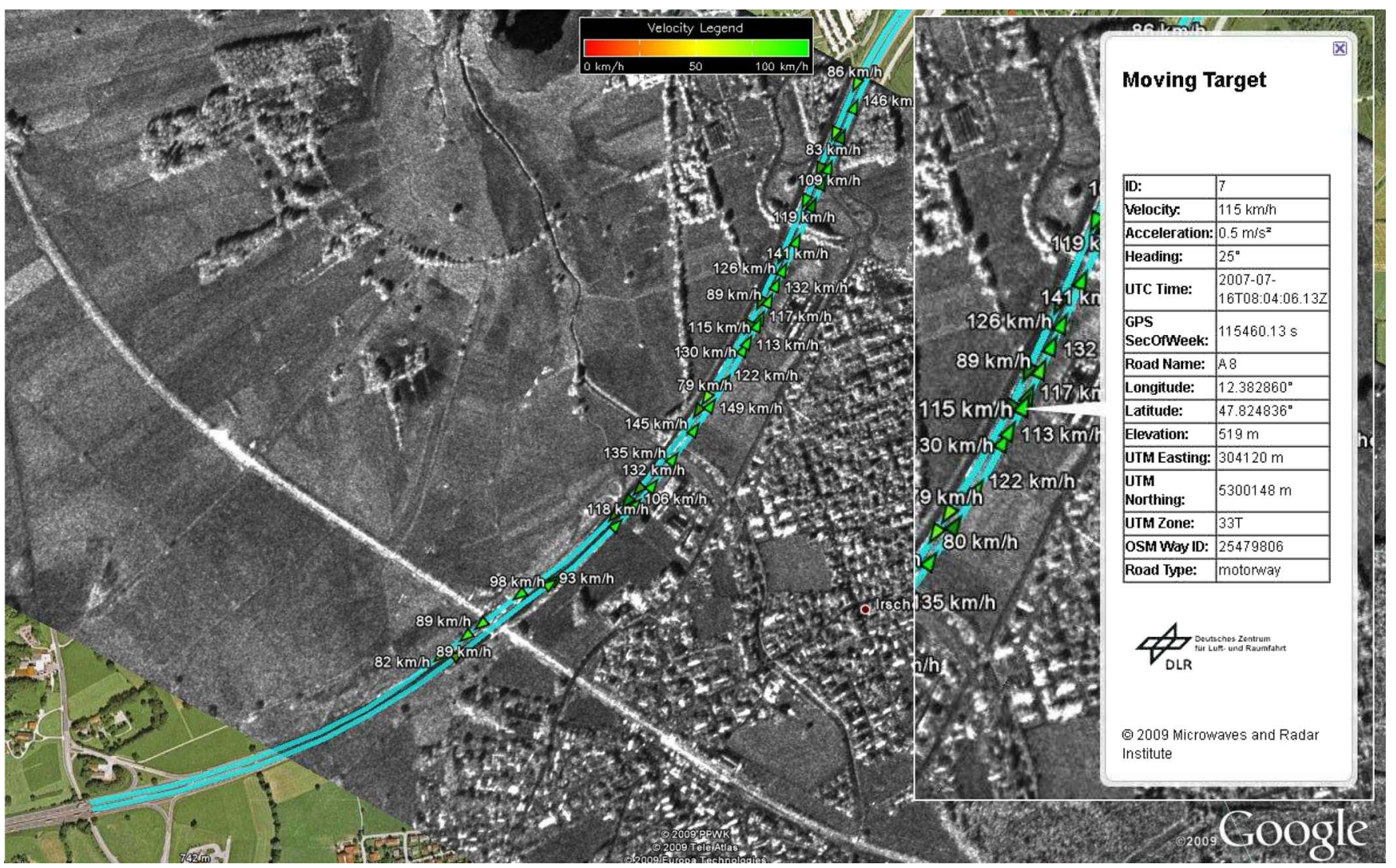

Fig. 21. Google Earth image overlaid with a single-channel SAR image acquired with F-SAR (image not processed with full quality, image size $2.0 \times 2.8 \mathrm{~km}$, data take rc07trmrad0302x1). The shown vehicles (color coded triangles) on the autobahn A8 near Chiemsee were automatically detected, and their parameters were automatically estimated using the proposed GMTI algorithm.

migration. For verifying the resolution of such ambiguities the same data as shown in Figs. 15 and 16 is used, but now decimated by a factor of two. That means, that the signal of the vehicle moving with about $84 \mathrm{~km} / \mathrm{h}$ is already backfolded (aliased) in Doppler as depicted in Fig. 18, right.

For resolving the Doppler ambiguity, the technique presented in Section III-G can be used. Different Doppler shifts $f_{\mathrm{DC}}$ and, hence, range migration slopes are assumed. For each Doppler shift assumption at each azimuth position, neighboring range samples around the "expected" range are taken, as shown in Fig. 19(a) and (b) for an array size of 1024 azimuth and 6 range samples. Afterward, for the obtained range/azimuth arrays (one array for each Doppler shift assumption), the total energy along each azimuth line is taken. The maximum energy indicates the correct range/azimuth array and hence the correct Doppler shift [cf. Fig. 19(b)], where the maximum energy along a single azimuth line is larger than in (a). The knowledge of the correct Doppler shift allows for unambiguous computation of the vehicle velocity (cf. Fig. 20, right). Due to the decimation, the azimuth ambiguities increase, and the clutter suppression performance decreases. Hence, the estimated velocities differ slightly (in this particular case about $\pm 1 \mathrm{~km} / \mathrm{h}$ ) from the estimates in the oversampled case shown in Fig. 15.

Furthermore, the range history of the moving vehicle signal can be aligned along range (by shifting each range line depending on its maximum; only reasonable if the vehicle size is not larger than the resolution), and the Doppler slope can be estimated using for example the "adaptive deramping" procedure [cf. Fig. 19(c)]. Once the Doppler slope is known, also the acceleration of the vehicle can be computed using (28). The whole image patch containing the vehicle signal can be refocused by removing the quadratic phase error [cf. Fig. 19(d)]. By considering more than 1024 azimuth samples, the azimuth resolution can be improved. Thus, it even might be possible to estimate the size and the shape of the moving vehicle, so that under circumstances, a discrimination between conventional passenger cars and large trucks becomes feasible. However, for a robust discrimination, more sophisticated inverse SAR imaging algorithms should be used [33].

In Fig. 21, a larger scene acquired over the Chiemsee region is shown. The detected moving vehicles are depicted as colored triangles pointing in the moving direction. The considered roads are shown in light blue. Two receiving channels were used for data acquisition. The average Doppler centroid is $415 \mathrm{~Hz}$, and the squint angle is $4.2^{\circ}$. Again, only a PRF of $2500 \mathrm{~Hz}$ was used. Thus, a lot of the vehicles moving on the autobahn A8 are ambiguous in Doppler. Nevertheless, by considering again the range migration, many of the ambiguities can be resolved, and additionally, by estimating the DOA angle using (71), some false detections can be reduced. Unfortunately, no ground truth data were available for the scene shown in Fig. 21. Thus, it is not possible to determine the probability of detection, the false alarm rate, and the velocity estimation errors for this scene. However, the estimated 

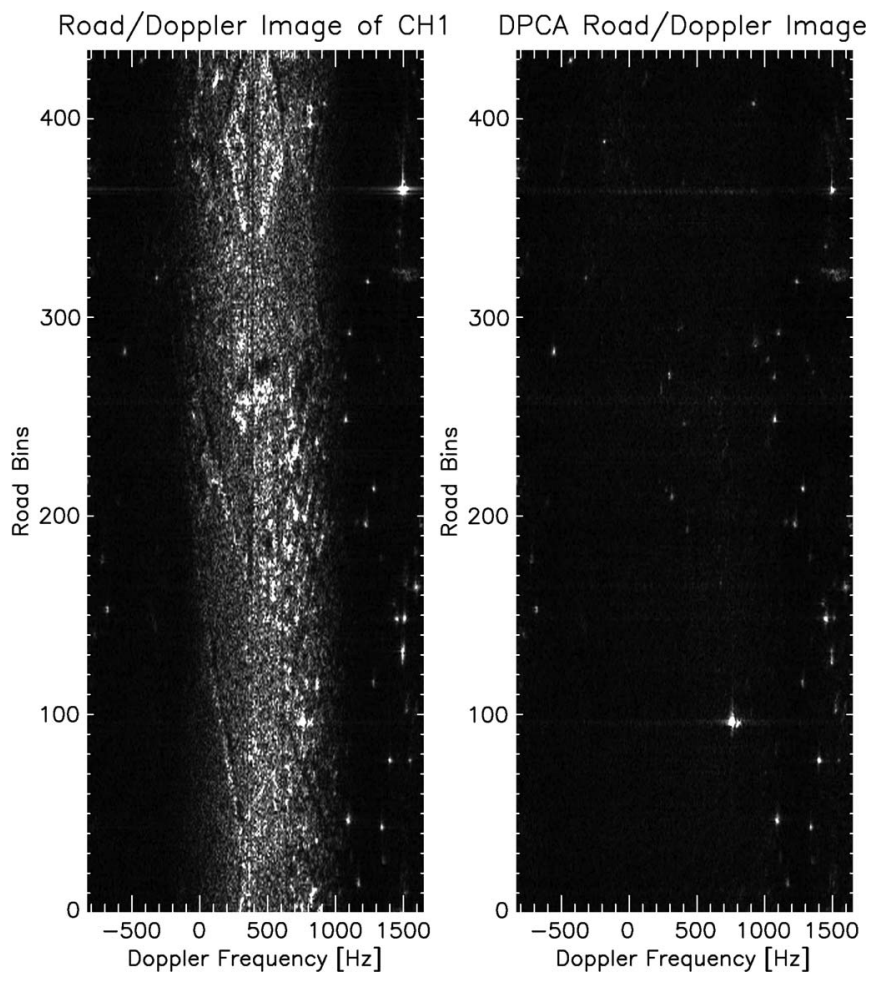

Fig. 22. Single-channel road/Doppler image (left) and clutter suppressed dual-channel DPCA road/Doppler image (right) of a road section of the autobahn A8. Even in the single-channel image, the fast moving vehicles falling outside the clutter band are clearly visible and detectable.

velocities, which are in the range from 79 to $149 \mathrm{~km} / \mathrm{h}$, are rather reasonable for a typical German autobahn.

If only a single-channel system is available, for fast moving vehicles, a detection and, hence, a motion and position estimation using the proposed algorithm is possible as shown in Fig. 22, left. Here, a road/Doppler image of a road section of the autobahn A8 is shown. The fast moving vehicle signals clearly can be seen outside the clutter band. The road/Doppler image is quite similar to a range/Doppler image, but instead of Fourier transformed azimuth signals of different ranges, the Fourier transformed azimuth signals around the different observed road points are plotted.

\section{CONCLusion}

A fast, real-time capable GMTI algorithm based on a priori knowledge, suitable for single- and multichannel radar and SAR data was presented. The algorithm was verified using real dual-channel SAR data acquired with DLR's F-SAR system. The obtained performance implies that the algorithm is suitable for real-time traffic monitoring applications. Although only dual-channel results using DPCA as clutter suppression technique were presented, the algorithm has the capability to be combined with more sophisticated techniques (e.g., STAP) for improving the overall performance. For instance, also modern multichannel airborne radar systems particularly designed for GMTI, like AER-II [12] and PAMIR [34], could benefit from the proposed algorithm, at least for real-time road traffic monitoring applications where vehicles moving on open land are not of particular interest. However, additional investigations are necessary to explore the full real-time traffic monitoring capabilities that may arise from an appropriate combination of STAP-like techniques with the proposed algorithm.

\section{ACKNOWLEDGMENT}

The authors would like to thank K.-H. Bethke, R. Scheiber, J. Fischer, and the group of A. Nottensteiner, all DLR. Without their efforts, particularly in F-SAR hardware and software development, it would not have been possible to successfully conduct the important GMTI experiments in 2007. The authors furthermore thank the anonymous reviewers for their valuable comments and suggestions which helped to improve the paper.

\section{REFERENCES}

[1] W.-Q. Wang, "Near-space vehicle-borne SAR with reflector antenna for high-resolution and wide-swath remote sensing," IEEE Trans. Geosci. Remote Sens., vol. 50, no. 2, pp. 338-348, Feb. 2012.

[2] S. Barbarossa, "Detection and imaging of moving objects with synthetic aperture radar-Part 1: Optimal detection and parameter estimation theory," Proc. Inst. Elect. Eng. F-Radar signal Process., vol. 139, no. 1, pp. 79-88, Feb. 1992

[3] B. Friedlander and B. Porat, "VSAR: A high resolution radar system for detection of moving targets," Proc. Inst. Elect. Eng. F-Radar, Sonar Navigat., vol. 144, no. 4, pp. 205-218, Aug. 1997.

[4] R. P. Perry, R. C. DiPietro, and R. L. Fante, "SAR imaging of moving targets," IEEE Trans. Aerosp. Electron. Syst., vol. 35, no. 1, pp. 188-200, Jan. 1999.

[5] C. H. Gierull and I. C. Sikaneta, "Raw data based two-aperture SAR ground moving target indication," in Proc. IEEE IGARSS, Toulouse, France, Jul. 2003, vol. 2, pp. 1032-1034.

[6] A. Budillon, G. Ferraiuolo, V. Pascazio, and G. Schirinzi, "Multichannel SAR interferometry via classical and Bayesian estimation techniques," EURASIP J. Appl. Signal Process., vol. 2005, no. 20, pp. 3180-3193, Jan. 2005.

[7] R. Klemm, Space-Time Adaptive Processing: Principles and Applications. London, UK: Inst. Elect. Eng., 1998.

[8] F. Meyer, S. Hinz, A. Laika, D. Weihing, and R. Bamler, "Performance analysis of the TerraSAR-X traffic monitoring concept," ISPRS J. Photogramm. Remote Sens., vol. 61, no. 3/4, pp. 225-242, Dec. 2006.

[9] M. Gabele and I. Sikaneta, "Motion parameter estimation of Dopplerambiguous moving targets in SAR-GMTI," in Proc. IRS, Cologne, Germany, Sep. 2007.

[10] S. V. Baumgartner and G. Krieger, "A priori knowledge based GMTI algorithm for traffic monitoring applications," in Proc. 8th EUSAR, Aachen, Germany, Jun. 2010, pp. 469-472.

[11] S. V. Baumgartner and G. Krieger, "Real-time road traffic monitoring using a fast a priori knowledge based SAR-GMTI algorithm," in Proc. IEEE IGARSS, Honolulu, HI, Jul. 2010, pp. 1843-1846.

[12] J. H. G. Ender, "The airborne experimental multi-channel SAR system AER-II," in Proc. 1st EUSAR, Königswinter, Germany, Mar. 1996, pp. 49-52.

[13] C. H. Gierull, "Digital channel balancing of along-track interferometric SAR data," Defense R\&D Canada, Ottawa, ON, Canada, Mar. 2003, Tech. Memo. DRDC Ottawa TM 2003-024.

[14] F. R. Dickey and M. M. Sante, "Final report on anti-clutter techniques," General Electric Co., Heavy Military Electron. Dept., Syracuse, New York, Rep. No. R65EMH37, 1953.

[15] J. J. Sharma, "The influence of target acceleration on dual-channel SARGMTI (Synthetic Aperture Radar Ground Moving Target Indication) data," M.S. thesis, Univ. Calgary, Calgary, AB, Canada, Oct., 2004.

[16] S. V. Baumgartner and G. Krieger, "SAR traffic monitoring using timefrequency analysis for detection and parameter estimation," in Proc. IEEE IGARSS, Boston, MA, Jul. 2008, vol. 2, pp. II-25-II-28.

[17] K. Bethke, S. Baumgartner, M. Gabele, D. Hounam, E. Kemptner, D. Klement, G. Krieger, and R. Erxleben, "Air-and spaceborne monitoring of road traffic using SAR moving target indication-Project TRAMRAD," ISPRS J. Photogramm. Remote Sens., vol. 61, no. 3/4, pp. 243-259, Dec. 2006. 
[18] G. Palubinskas and H. Runge, "Radar signatures of a passenger car," IEEE Geosci. Remote Sens. Lett., vol. 4, no. 4, pp. 644-648, Oct. 2007.

[19] P. Lombardo, F. Colone, and D. Pastina, "Monitoring and surveillance potentialities obtained by splitting the antenna of the COSMO-SkyMed SAR into multiple sub-apertures," Proc. Inst. Elect. Eng.-Radar, Sonar Navigat., vol. 153, no. 2, pp. 104-116, Apr. 2006.

[20] J. H. G. Ender, C. H. Gierull, and D. Cerutti-Maori, "Improved spacebased moving target indication via alternate transmission and receiver switching," IEEE Trans. Geosci. Remote Sens., vol. 46, no. 12, pp. 39603974, Dec. 2008.

[21] I. G. Cumming and F. H. Wong, Digital Signal Processing of Synthetic Aperture Radar: Algorithms and Implementation. Norwood, MA: Artech House, 2005.

[22] J. C. Curlander and R. N. McDonough, Synthetic Aperture Radar: Systems and Signal Processing. New York: Wiley, 1991.

[23] R. K. Raney, "Synthetic aperture imaging radar and moving targets," IEEE Trans. Aerosp. Electron. Syst., vol. AES-7, no. 3, pp. 499-505, May 1971.

[24] J. Jao, "Theory of synthetic aperture radar imaging of a moving target," IEEE Trans. Geosci. Remote Sens., vol. 39, no. 9, pp. 1984-1992, Sep. 2001.

[25] J. Radon, "Über die Bestimmung von Funktionen durch ihre Integralwerte längs gewisser Mannigfaltigkeiten," Berichte Sächsische Akademie der Wissenschaften, Leipzig, Mathematisch-Physikalische Klasse, vol. 69, pp. 262-277, 1917.

[26] P. V. C. Hough, "Method and means for recognizing complex patterns," US Patent 3069 654, Dec. 18, 1962.

[27] U. Nickel, "Overview of generalized monopulse estimation," IEEE Aerosp. Electron. Syst. Mag., vol. 21, pt. 2, no. 6, pp. 27-56, Jun. 2006.

[28] R. Horn, A. Nottensteiner, and R. Scheiber, "F-SAR-DLR's advanced airborne SAR system onboard DO228," in Proc. 7th Eur. Conf. Synthetic Aperture Radar, Friedrichshafen, Germany, Jun. 2008, pp. 1-4.

[29] S. Baumgartner, M. Gabele, N. Gebert, R. Scheiber, G. Krieger, K.-H. Bethke, and A. Moreira, "Digital beamforming and traffic monitoring using the new F-SAR system of DLR," in Proc. IRS, Cologne, Germany, Sep. 2007.

[30] OpenStreetMap-The Free Wiki World Map, Jul. 2009. [Online]. Available: http://www.openstreetmap.org

[31] Shuttle Radar Topography Mission-The Mission to Map the World, Jul. 2009. [Online]. Available: http://www2.jpl.nasa.gov/srtm/

[32] Google Earth2009, Ju.. [Online]. Available: http://earth.google.com/

[33] V. Chen and H. Ling, Time-Frequency Transforms for Radar Imaging and Signal Analysis. Norwood, MA: Artech House, 2002.

[34] D. Cerutti-Maori, J. Klare, A. R. Brenner, and J. G. H. Ender, "Wide area traffic monitoring with the SAR/GMTI system PAMIR," IEEE Trans. Geosci. Remote Sens., vol. 46, no. 10, pp. 3019-3030, Oct. 2008.

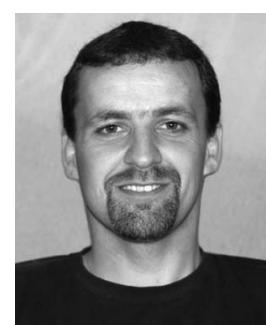

Stefan V. Baumgartner (GSM'10) received the Dipl.-Ing. degree in electrical engineering and communication technology from the Graz University of Technology, Graz, Austria in 2004.

Since 2004, he has been with the Microwaves and Radar Institute (HR), German Aerospace Center (DLR), Oberpfaffenhofen, Germany. He is currently with the Radar Concepts Department, where his field of activity is the development of ground moving target indication and parameter estimation algorithms for future road-traffic-monitoring applications using multichannel air- and spaceborne synthetic aperture radars (SAR). His research interests include SAR along-track interferometry, time-frequency analysis, and other advanced signal and imaging processing techniques.

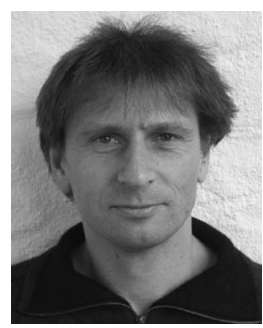

Gerhard Krieger (M'04-SM'10) received the Dipl.-Ing. (M.S.) and Dr.-Ing. (Ph.D.) degrees (with honors) in electrical and communication engineering from the Technical University of Munich, Munchen, Germany, in 1992 and 1999, respectively.

From 1992 to 1999 , he was with the LudwigMaximilians University, Munich, where he conducted multidisciplinary research on neuronal modeling and nonlinear information processing in biological and technical vision systems. In 1999, he joined the Microwaves and Radar Institute (HR) of the German Aerospace Center (DLR), Oberpfaffenhofen, Germany, where he developed signal and image processing algorithms for a novel forward looking radar system employing digital beamforming on receive. From 2001 to 2007, he led the new synthetic aperture radar (SAR) Missions Group which pioneered the development of advanced bistatic and multistatic radar systems as exemplified by the forthcoming TanDEM-X mission as well as innovative multichannel SAR techniques and algorithms for high-resolution wide-swath SAR imaging. Since 2008, he has been Head of the new Radar Concepts Department of the Microwaves and Radar Institute, DLR. He is author of more than 40 peer-reviewed journal papers, four invited book chapters, about 250 conference papers, and five patents. His current research interests focus on the development of multichannel radar techniques and algorithms for innovative multiple-input-multiple-output (MIMO) SAR systems, the demonstration of novel interferometric and tomographic earth observation applications, and the conceptual design of advanced bi- and multistatic radar missions.

Dr. Krieger received several national and international awards, including the W.R.G. Baker Prize Paper Award from the IEEE Board of Directors and the IEEE Transactions Prize Paper Award of the Geoscience and Remote Sensing Society. 